\title{
Random dynamic load identification for stochastic structural-acoustic system using an adaptive regularization parameter and evidence theory
}

\author{
Z.C. $\mathrm{He}^{1}$, Zhuomin Zhang ${ }^{1}$, Eric $\mathrm{Li}^{2 *}$ \\ ${ }^{1}$ State Key Laboratory of Advanced Design and Manufacturing for Vehicle Body, Hunan University, Changsha, \\ 410082 P. R. China \\ ${ }^{2}$ School of Science, Engineering \& Design, Teesside University, Middlesbrough, UK
}

\begin{abstract}
In practical engineering, the non-contact measurement is more excellent due to preventing the potential damage of contact transducers. In this paper, a sound pressure-based method is proposed for identifying the random dynamic load acting on structural-acoustic coupled (SAC) system. Considering the characteristic of pseudo excitation and the influence rule of the regularization parameter, an adaptive regularization parameter is formulated to deal with the ill-posedness and reduce the error amplification of random excitation identification. Compared with the Tikhonov regularization method and Moore-Penrose pseudo inverse (MPI) method, the proposed improved regularization method reduces the identified error by $2.24 \times 10^{3} \mathrm{~N}^{2} / \mathrm{Hz}$ and $2.91 \times 10^{5} \mathrm{~N}^{2} / \mathrm{Hz}$ at $4 \mathrm{~Hz}$ and $6 \mathrm{~Hz}$ respectively in the studied example. The identified loads by improved regularization method match the actual loads adequately in the full frequency. Furthermore, owing to the uncertainty of the SAC system, the method for solving the stochastic system problem is investigated by the evidence theory combined with the interval analysis. Accordingly, the lower and upper bounds are derived to assess the statistical property of identified random dynamic loads. Finally, the effectiveness of the proposed methods is demonstrated by several numerical examples of complex stochastic systems.
\end{abstract}

Key words: random dynamic loads identification; structural-acoustic coupling; stochastic systems; improved regularization method; evidence theory

\footnotetext{
* Corresponding author. Tel./fax: +86 73188822051.

E-mail address: q.li@tees.ac.uk; ericsg2012@gmail.com
} 


\section{Introduction}

Random dynamic loads exist in reality commonly in the engineering field, such as road loads imposed on moving vehicle, wave loads applied to the offshore structure and wind loads acting on tall building $[1,2]$. The various kinds of random dynamic loads play an important role in reliability evaluation and NVH (Noise, Vibration and Harshness) analysis for engineering structures $[3,4]$. Thus, determining the random excitations exactly is a necessary and momentous task. Unfortunately, measuring random dynamic loads directly is very difficult and impractical owing to the limitation of economic condition and engineering technology. In contrast, there is an indirect way that can be applied to identify conveniently the random excitations based on the measurement of dynamic responses.

After rapid development in the last several decades, the technologies for identifying dynamic forces indirectly have been investigated extensively, which can be divided into time-domain [5-7] and frequency-domain load identification methods [8,9]. As early as 1979, Bartlett et al. [10] evaluated the lateral and vertical hub excitations acting on a helicopter model applying the least-square scheme, which is one of the most extensively used method among the conventional load identification methods. Karlsson [11] applied the measurement of accelerations and strain responses to identify the external loads on an existing vibrating structure. Based on structural displacements, Liu et al. [12] proposed the inverse methods to determine the loads on composite laminates in time domain. In sum, the above-mentioned researches mainly employed the vibration-based responses usually measured in a contact way, such as accelerations, velocities, displacements and strains to 
identify the unknown loads. To receive the vibration signal of the structure, the attaching transducers are installed on the structure surface, which influence the behavior of the vibration structure. Furthermore, owing to the influence of the weight of the transducers, the vibration of the structure may be destroyed badly, especially the thin-walled structure.

In practical engineering applications, compared with the contact measurement, the non-contact measurement is more useful with advantages of appropriate operating condition and less potential destruction of measuring responses [13]. Lately, more and more researchers have been interested in the applications of the non-contact acoustic techniques, such as the determination of force distribution as well as force location and the vibration reconstruction of the SAC interface. Near-field acoustic holography is widely used in localization of sound source and accurate determination of vibration on SAC interface [14-16]. Moreover, Lee and Xu [17] applied the acoustic transfer matrix method to predict the transmission loss of acoustic materials. Deng et al. [18] identified the interior and exterior sound source and analyzed the acoustic contribution for the car on the basis of the acoustic array technology. In the present study, based on the non-contact measurement, a novel sound pressure-based method is proposed for identifying the random dynamic load acting on SAC system.

The above-mentioned methods for random excitation identification are mostly investigated on those structures with deterministic parameters. Nevertheless, a complex SAC system often involves numerous uncertainties, such as the model manufacturing error, the hostile environment factors and the erratic loading conditions. In dealing with the 
dynamic load identification problems for SAC system with uncertainties, the Monte Carlo simulation (MCS) method [19, 20], which is usually perceived as reference method, can provide very accurate solutions at the expenses of low computational efficiency. Liu and Sun [21, 22] applied matrix perturbation method and Gegenbauer polynomial expansion theory to identify dynamic forces imposed on uncertain structures in time domain. Matrix perturbation method $[23,24]$ and orthogonal expansion method $[25,26]$ are both related to convert the random parameters function into power series form through approximate first-order Taylor series expansion. If the uncertainties of the parameters are fairly large, the response region obtained using the first-order Taylor analysis method would fail to contain the real region of the dynamic response of uncertain structures. Therefore, the second-order analysis method should be considered. However, the second-order analysis method related to operations of interval may result in an exorbitantly overestimated dynamic response region, which makes the result useless for practical engineering problems.

In the present study, the evidence theory is combined with interval analysis. By discretizing the PDF of each random parameter into several subintervals, the variations of random parameters in each subinterval are reduced. With the increase of the number of discrete intervals in the random sample space, the assumption of first-order Taylor expansion is gradually enhanced. For this reason, aimed at the SAC system containing random parameter with large coefficient of variation and multimodal distribution, a novel random load identification method is developed based on the evidence theory and the interval analysis method, with advantages of enormous applications and more accurate 
inverse solutions. The evidence theory method was first proposed by Dempster in 1967 [27], and later it was further studied and developed by Shafer [28]. Thus, the evidence theory is called Dempster-Shafer theory or D-S evidence theory, which has formed a more complete theoretical system. Mourelatos and Zhou [29] put forward an efficient reliability analysis method based on evidence theory. Agarwal et al. [30] and Alyanak et al. [31] used the sequence approximate optimization strategy and the gradient mapping method to develop a reliability optimization design method based on the evidence theory. Jiang and Bai [32-36] combined the evidence theory with the finite element method, developed an uncertain structural analysis method based on the evidence theory, and introduced the moment concept of the probability theory to calculate the moments of evidence variable and the moments of evidence function through interval arithmetic.

In addition, in the processes of inversion for load identification, the ill-posed problem is also an important issue that must be solved. Okubo [37] investigated the effect of the measurement noise on the inversion process. Due to the ill-conditioned frequency response function (FRF) matrix at frequencies at the structural resonances, the noise involving in measurement responses, even a minor error, can result in highly ill-posedness and destroy badly the stability and accuracy of identifying loads. Consequently, various approaches have been developed to solve the ill-posedness and increase the identification accuracy [38]. Based on the total least-squares (TLS) method and enhanced least squares, Liu et al. [39] reduced the effects of measurement noise and identified the dynamic loads effectively in frequency domain. Using the generalized Tikhonov regularization methods, 
Aucejo [40, 41] introduced prior information on the measured noise as well as on the excitation characteristics to determine the sparsity or regularity. Liu et al. [42] developed an efficient method to reduce the ill-posedness by formulating a novel global kernel functions matrix so as to determine the dynamic loads accurately and stably. Zhang and Antoni [43] applied the Bayesian method to reconstruct the dynamic force and derive the Bayesian credible intervals in consideration of model uncertainty and measurement noise. On the basis of weighted total least squares method and weighted Tikhonov regularization method, Jia and Yang $[44,45]$ performed the experimental method to identify the random excitation taking into account the measurement error of FRF and responses. However, the most of the above-mentioned methods concentrate on the load identification in time domain or deterministic load identification in frequency domain. Considering the random excitation identification and SAC system, a novel improved regularization method is developed to solve the ill-posedness and decrease the error propagation of the random dynamic loads to be identified. According to the features of pseudo excitation as well as the impact of the regularization parameter on solution norm and residual norm, the improved regularization parameter is proposed for raising the stability and the accuracy of identifying random dynamic forces. In comparison with Moore-Penrose pseudo inverse (MPI) method and Tikhonov regularization method, the proposed improved regularization method achieves more accurate and stable identified loads.

The rest of paper is organized as follows: in Section 2, the basic theory of SAC system is briefly introduced and the forward problem of random excitation identification using the 
sound pressure is described. Section 3 introduces the evidence theory combined with interval analysis to solve the structural uncertainty problem. Section 4 proposes an improved regularization method to handle the ill-posed problems. Finally, Section 5 illustrates the effectiveness and feasibility of the proposed approaches on several numerical examples and the conclusions are given in Section 6.

\section{Formulation of forward problem for SAC system}

As shown in Fig. 1, the SAC system consists of structural domain $\Omega_{\mathrm{s}}$, acoustic domain $\Omega_{\mathrm{a}}$ and coupled boundary $\Gamma_{\mathrm{sa}}$. Therein, the fluid domain boundary is surrounded by the flexible structural plate and acoustic boundary $\Gamma_{a}$, and the boundary conditions of structural domain are composed by displacement boundary condition $\Gamma_{\mathrm{s}}$ and force boundary condition $\Gamma_{\mathrm{f}}$. The present study is based on the weak coupling system. In the weak coupling problems, the frequency response of the pressure is obtained and the random dynamic load identification can be achieved based on the acoustic pressure responses. As the acoustic pressure acting on the structure is almost negligible, the strong coupling system is not considered in this work. 


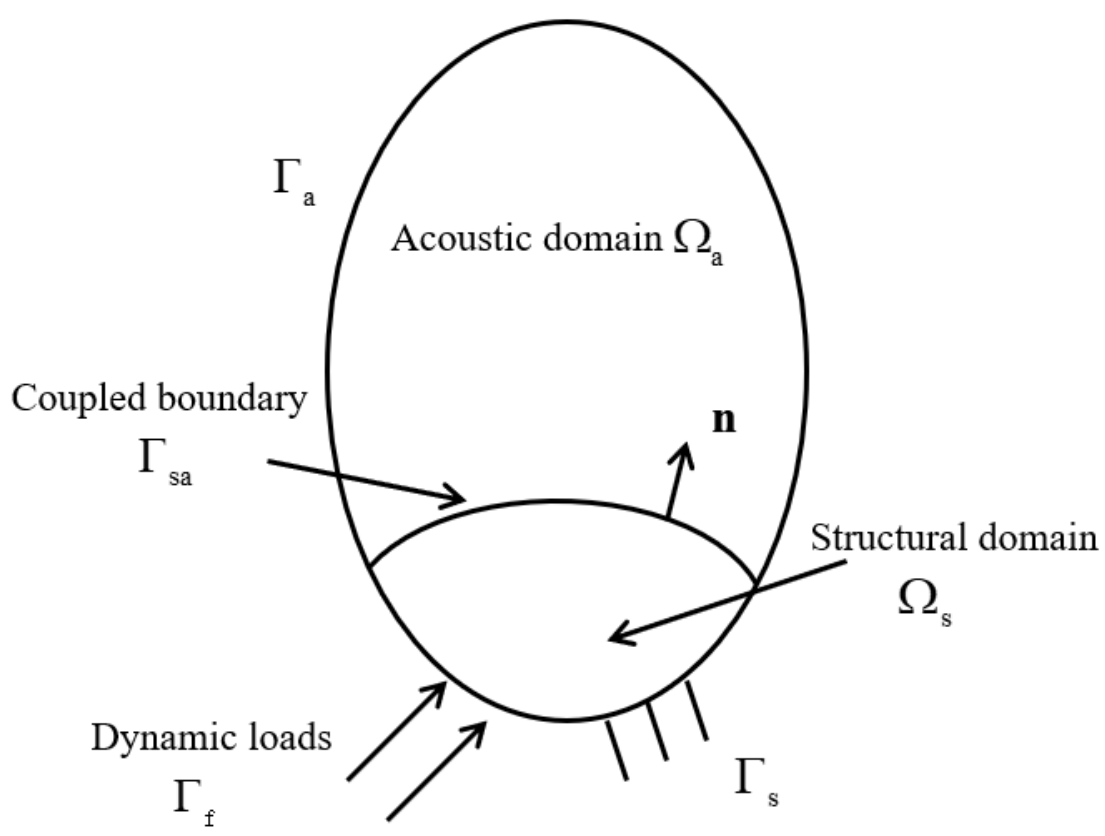

Fig. 1. Schematic diagram of SAC system.

\subsection{Structural dynamic model subjected to random excitation}

With respect to the linear time-invariant deterministic structural domain excited by stationary random excitations in the SAC system, the power spectral density (PSD) of the structural vibration responses on the coupled boundary can be expressed as follows:

$$
\mathbf{S}_{\mathbf{Y Y}}(\omega)=\mathbf{H}_{\mathrm{s}}(\omega) \mathbf{S}_{\mathbf{F F}}(\omega) \mathbf{H}_{\mathrm{s}}(\omega)^{H}
$$

in which $\mathbf{S}_{\mathbf{Y Y}}(\omega) \in \mathbf{C}^{m \times m}$ and $\mathbf{S}_{\mathbf{F F}}(\omega) \in \mathbf{C}^{n \times n}$ are the dynamic vibration responses PSD matrix and PSD matrix of random excitations, respectively. $\mathbf{H}_{\mathrm{s}}(\omega) \in \mathbf{C}^{m \times n}$ represents the FRF matrix from exciting positions on structural domain to the coupled boundary. The superscript $H$ is referred to the complex conjugate transpose operator, and $\mathbf{C}^{m \times m}$ means that the rows and columns of the matrix are $m$ and $m$.

In the structural computation of the finite element discrete system, the finite element method of the structural domain can be described in the following form: 


$$
\mathbf{M}_{\mathrm{s}} \ddot{\mathbf{y}}(t)+\mathbf{C}_{\mathrm{s}} \dot{\mathbf{y}}(t)+\mathbf{K}_{\mathrm{s}} \mathbf{y}(t)=\mathbf{f}(t)
$$

in which $\ddot{\mathbf{y}}(t), \dot{\mathbf{y}}(t)$ and $\mathbf{y}(t)$ denote the acceleration vector, velocity vector and displacement vector of the structure responses on coupled boundary, respectively. $\mathbf{M}_{\mathrm{s}}, \mathbf{C}_{\mathrm{s}}$ and $\mathbf{K}_{\mathrm{s}}$ are the structure mass matrix, damping matrix and structure stiffness matrix, respectively. $t$ represents the time.

$$
\mathbf{M}_{\mathrm{s}}=\int_{\Omega_{\mathrm{s}}} \rho_{\mathrm{s}} \mathbf{N}_{\mathrm{s}}^{H} \mathbf{N}_{\mathrm{s}} \mathrm{d} \Omega, \quad \mathbf{C}_{\mathrm{s}}=\int_{\Omega_{\mathrm{s}}} \mathbf{N}_{\mathrm{s}}^{H} \mathbf{N}_{\mathrm{s}} \varsigma \mathrm{d} \Omega, \quad \mathbf{K}_{\mathrm{s}}=\int_{\Omega_{\mathrm{s}}} \mathbf{B}_{\mathrm{s}}^{H} \mathbf{D}_{\mathrm{s}} \mathbf{B}_{\mathrm{s}} \mathrm{d} \Omega
$$

where $\mathbf{N}_{\mathrm{s}}, \rho_{\mathrm{s}}$ stand for the structure shape function and the structure density, respectively; $\mathbf{D}_{\mathrm{s}}, \mathbf{B}_{\mathrm{s}}$ and $\varsigma$ represent the structure elastic matrix, the structure strain matrix and the damping factor, respectively.

$\mathbf{f}(t) \in \mathbf{C}^{n \times 1}$ denotes the random dynamic load vector acting on the structural domain, and the derived PSD matrix $\mathbf{S}_{\mathbf{F F}}(\omega)$ is known. According to the characteristic of non-negative definite Hermit matrix, the PSD matrix can be decomposed as follows:

$$
\mathbf{S}_{\mathbf{F F}}(\omega)=\sum_{j=1}^{r} \sigma_{j} \boldsymbol{\varphi}_{j} \boldsymbol{\varphi}_{j}{ }^{H}=\sum_{j=1}^{r} \tilde{\mathbf{F}}_{j}(\omega) \tilde{\mathbf{F}}_{j}(\omega)^{H} \quad(r \leq n)
$$

where $\boldsymbol{\varphi}_{j}$ and $\sigma_{j}$ are eigenvector and corresponding eigenvalue of PSD matrix $\mathbf{S}_{\mathbf{F F}}(\omega)$; $r$ denotes the rank of $\mathbf{S}_{\mathbf{F F}}(\omega)$. Obviously, $\tilde{\mathbf{F}}_{j}(\omega)=\sqrt{\sigma_{j}} \boldsymbol{\varphi}_{j}$ represents the $j$-order pseudo excitation and the superscript $\sim$ is known as the pseudo variable.

Based on that, we can formulate $r$ mutually independent pseudo harmonic dynamic loads as follows[46]:

$$
\tilde{\mathbf{f}}_{j}(t)=\tilde{\mathbf{F}}_{j}(\omega) e^{i \omega t}=\sqrt{\sigma_{j}} \boldsymbol{\varphi}_{j} e^{i \omega t} \quad(j=1,2, \ldots, r)
$$

Substituting Eq. (5) into Eq. (2), the complicated random vibration system of the structural domain is converted into a straightforward harmonic equation of motion. 
Moreover, on the basis of modal superposition method, the pseudo harmonic vibration responses are obtained

$$
\tilde{\mathbf{y}}_{j}(t)=\tilde{\mathbf{Y}}_{j}(\omega) e^{i \omega t} \quad(j=1,2, \ldots, r)
$$

where $\tilde{\mathbf{Y}}_{j}(\omega)=\mathbf{H}(\omega) \tilde{\mathbf{F}}_{j}(\omega)$ denotes the $j$-order pseudo vibration response.

\subsection{Forward problem of random excitation identification for SAC system}

With regard to the acoustic domain in the SAC system, its discrete system equation can be expressed as follows[47]:

$$
\mathbf{M}_{\mathrm{a}} \ddot{\mathbf{p}}+\mathbf{C}_{\mathrm{a}} \dot{\mathbf{p}}+\mathbf{K}_{\mathrm{a}} \mathbf{p}=\mathbf{f}_{\mathrm{sa}}
$$

in which $\mathbf{K}_{\mathrm{a}}, \mathrm{C}_{\mathrm{a}}$ and $\mathbf{M}_{\mathrm{a}}$ denote the acoustic stiffness matrix, acoustic damping matrix and acoustic mass matrix, respectively. $\mathbf{p}$ and $\mathbf{f}_{\mathrm{sa}}$ stand for the acoustic pressure responses vector in the acoustic domain and the vector of acoustic loads, respectively.

$$
\mathbf{K}_{\mathrm{a}}=\int_{\Omega_{\mathrm{a}}}\left(\nabla \mathbf{N}_{\mathrm{a}}\right)^{H} \nabla \mathbf{N}_{\mathrm{a}} \mathrm{d} \Omega=\int_{\Omega_{\mathrm{a}}} \mathbf{B}_{\mathrm{a}}^{H} \mathbf{B}_{\mathrm{a}} \mathrm{d} \Omega, \quad \mathbf{M}_{\mathrm{a}}=\int_{\Omega_{\mathrm{a}}} \mathbf{N}_{\mathrm{a}}^{H} \mathbf{N}_{\mathrm{a}} \mathrm{d} \Omega
$$

Owing to the continuity of the acoustic pressure and the structure displacement on the coupled boundary in the SAC system, the acoustic loads vector can be deduced as:

$$
\mathbf{f}_{\mathrm{aq}}=\int_{\Gamma_{\mathrm{sa}}} \mathbf{N}_{\mathrm{a}}^{H} \rho_{\mathrm{a}} \ddot{\mathbf{y}}_{\mathrm{a}} \mathrm{d} \Gamma=\rho_{\mathrm{a}}\left(\int_{\Gamma_{\mathrm{sa}}} \mathbf{N}_{\mathrm{a}}^{H} \mathbf{n}_{\mathrm{a}} \mathbf{N}_{\mathrm{s}} \mathrm{d} \Gamma\right) \ddot{\mathbf{y}}
$$

in which $\mathbf{n}_{\mathrm{a}}$ represents the normal direction of the coupled boundary. With regard to the weak coupled system regardless of the influence of acoustic pressure upon structural system, the SAC equation can be derived by combining the Eq. (2) and Eq. (7).

$$
\left[\begin{array}{cc}
\mathbf{M}_{\mathrm{s}} & 0 \\
\rho_{\mathrm{a}} \mathbf{G}^{\mathrm{T}} & \mathbf{M}_{\mathrm{a}}
\end{array}\right]\left\{\begin{array}{c}
\ddot{\mathbf{y}} \\
\ddot{\mathbf{p}}
\end{array}\right\}+\left[\begin{array}{cc}
\mathbf{C}_{\mathrm{s}} & 0 \\
0 & \mathbf{C}_{\mathrm{a}}
\end{array}\right]\left\{\begin{array}{c}
\dot{\mathbf{y}} \\
\dot{\mathbf{p}}
\end{array}\right\}+\left[\begin{array}{cc}
\mathbf{K}_{\mathrm{s}} & -\mathbf{G} \\
0 & \mathbf{K}_{\mathrm{a}}
\end{array}\right]\left\{\begin{array}{l}
\mathbf{y} \\
\mathbf{p}
\end{array}\right\}=\left\{\begin{array}{l}
\mathbf{f} \\
0
\end{array}\right\}
$$

in which $\mathbf{G}$ denotes the coupled matrix on the interface: 


$$
\mathbf{G}=\int_{\Gamma_{\mathrm{sa}}} \mathbf{N}_{\mathrm{a}}^{H} \mathbf{n}_{\mathrm{a}} \mathbf{N}_{\mathrm{s}} \mathrm{d} \Gamma
$$

In the coupled system solution, the dynamic excitation is applied to the structural domain, and the sound pressure response is generated by the vibration of the structure through the coupled boundary. The response in the acoustic domain in Eq. (7) can be rewritten as follows:

$$
\mathbf{M}_{\mathrm{a}} \ddot{\mathbf{p}}+\mathbf{C}_{\mathrm{a}} \dot{\mathbf{p}}+\mathbf{K}_{\mathrm{a}} \mathbf{p}=\rho_{\mathrm{a}} \mathbf{G}^{H} \ddot{\mathbf{y}}
$$

where $\mathbf{y}=\left\{y_{1}, y_{2}, \ldots, y_{i}, \ldots, y_{N}\right\}^{H}$, and $y_{i}$ represents the structural vibration response of the $i$-th node on the coupled interface.

Combining Eq. (6) and Eq. (12), based on the modal superposition theory, the pseudo harmonic acoustic pressure response can be further obtained as follows:

$$
\begin{gathered}
\tilde{\mathbf{p}}_{j}(t)=\tilde{\mathbf{P}}_{j}(\omega) e^{i \omega t} \quad(j=1,2, \ldots, r) \\
\tilde{\mathbf{P}}_{j}(\omega)=\mathbf{T}(\omega) \tilde{\mathbf{F}}_{j}(\omega)
\end{gathered}
$$

where $\tilde{\mathbf{P}}_{j}(\omega)$ denotes the pseudo acoustic pressure response in the acoustic domain, and $\tilde{\mathbf{p}}_{j}(t)$ is the corresponding pseudo harmonic acoustic pressure in the time domain; $\mathrm{T}(\omega)$ stands for the FRF matrix from exciting positions on structural domain to the measurement points of acoustic pressure response.

Thus, the PSD matrix of acoustic pressure response can be derived

$$
\mathbf{S}_{\mathbf{P P}}(\omega)=\sum_{j=1}^{r} \tilde{\mathbf{P}}_{j}(\omega) \tilde{\mathbf{P}}_{j}(\omega)_{j}{ }^{H}
$$

From Eq. (14), it can be inferred that the acoustic pressure response can be acquired directly by the random dynamic loads in the structural domain. Therefore, according to the inverse pseudo excitation method [48], the random dynamic load identification can be 
achieved based on the measured acoustic pressure responses, which is different from the measurement of the structural vibration responses.

\section{Evidence theory for compound random load identification}

With regard to the SAC system containing random parameter with large coefficient of variation and multimodal distribution, a novel random load identification method is developed based on the evidence theory and the interval analysis method. The finite sample space of the random parameters is deemed as the frame of discernment (FD). The probability density function (PDF) of the random parameters is discretized into a number of subintervals that are regarded as the focal element (FE), and the probability of each subinterval is computed as the basic probability assignment (BPA) for each FE. Based on that, the problem of stochastic system is converted into an evidence analysis problem.

\subsection{Evidence model of random load identification for stochastic SAC system}

The FRF, representative of the system dynamic performance, would possess the random characteristic when some system properties such as material densities and Young's modulus are not completely determined. In this case, the identified random dynamic load is relevant to the random parameters of the coupled system instead of a definite result. Consequently, the identifying random excitation and FRF are expressed as the functions of frequency as well as stochastic parameters. The forward problem for the SAC system can be rewritten as

$$
\mathbf{S}_{\mathbf{P P}}(\omega)=\mathbf{T}(\omega, \mathbf{\kappa}) \mathbf{S}_{\mathbf{F F}}(\omega, \mathbf{\kappa}) \mathbf{T}(\omega, \mathbf{\kappa})^{H}
$$

where $\boldsymbol{\kappa}$ represents the $q$-dimensional stochastic parameters vector of the coupled system. 
Hence, the relation between pseudo acoustic pressure responses and pseudo excitations can be reformulated as

$$
\tilde{\mathbf{P}}_{j}(\omega)=\mathbf{T}(\omega, \mathbf{\kappa}) \tilde{\mathbf{F}}_{j}(\omega, \boldsymbol{\kappa})
$$

Discretizing the PDF of each random parameter $\kappa_{l}$ in $\boldsymbol{\kappa}$ into a number of subintervals, the random parameter of which is considered as evidence variable, the PDF of random parameters in the subintervals is regarded as the BPA of the evidence variable on the corresponding FE. As shown in Fig. $2, \xi_{j i}$ denotes the $i$-th FE of the $j$-th evidence variable. As a consequence, Eq. (15) and Eq. (16) can be rewritten as follows:

$$
\begin{gathered}
\tilde{\mathbf{P}}_{j}(\omega)=\mathbf{T}(\omega, \xi) \tilde{\mathbf{F}}_{j}(\omega, \xi) \\
\mathbf{S}_{\mathbf{P P}}(\omega)=\mathbf{T}(\omega, \xi) \mathbf{S}_{\mathbf{F F}}(\omega, \xi) \mathbf{T}(\omega, \xi)^{H}
\end{gathered}
$$

where $\xi$ stands for the $q$-dimensional evidence variables vector. The joint BPA of evidence variables can be derived by the BPA of each evidence variable. In the current studies of evidence theory, the evidence variables are generally considered as independent mutually. Thus, the joint FD $\Theta$ can be defined as follows [33]:

$$
\Theta=\xi_{1} \times \xi_{2} \cdots \xi_{q}=\left\{\mathbf{N}_{k}=\left[\zeta_{1}, \zeta_{2}, \cdots, \zeta_{q}\right], \zeta_{l} \in \xi_{l}, l=1,2, \cdots, q\right\}
$$

where $\mathbf{N}_{k}$ stands for the FE of joint FD $\Theta, \zeta_{l}$ represents the FE of $l$-th evidence variable. Hence, the joint BPA can be obtained

$$
\mathrm{B}\left(\mathbf{N}_{k}\right)=\left\{\begin{array}{cc}
\prod_{l=1}^{q} \mathrm{~B}\left(\zeta_{l}\right) & \\
0 & \text { others }
\end{array}\right.
$$




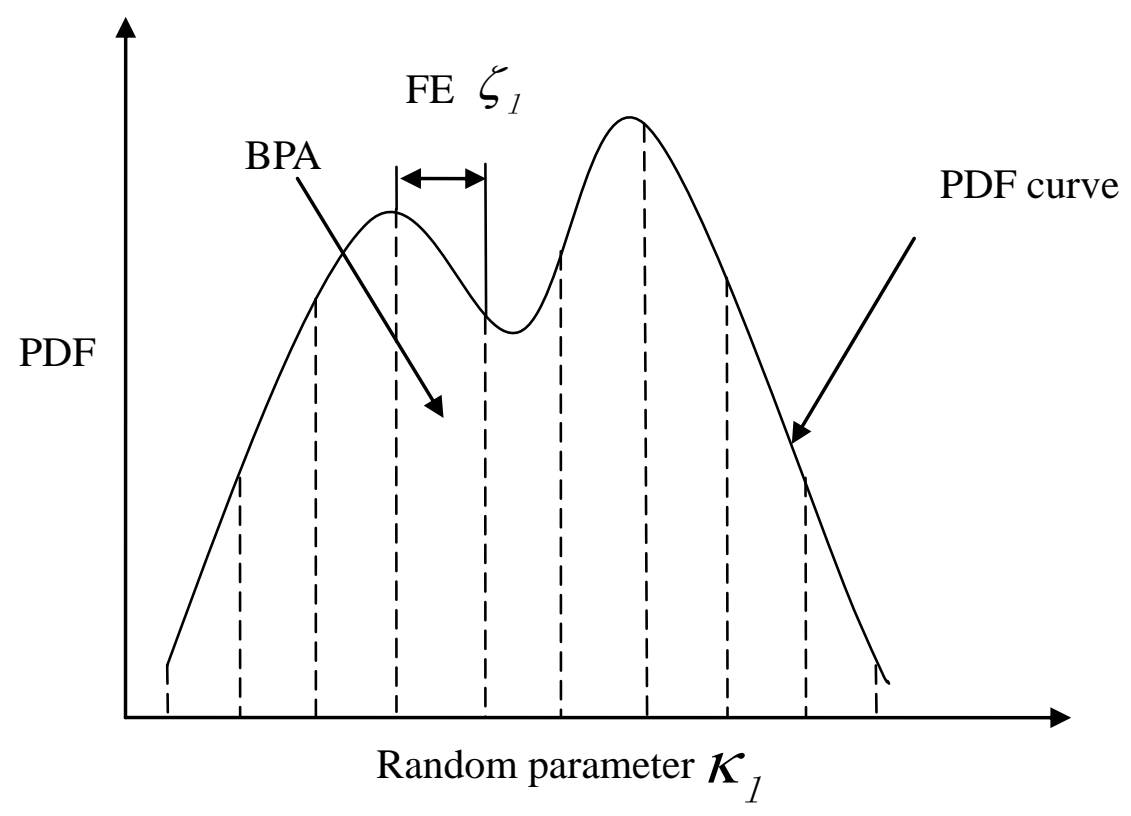

Fig. 2. Schematic diagram of discrete PDF.

For any FE $\mathbf{N}_{k}$ of joint FD, the forward problem of random excitation identification for stochastic SAC system can be expressed as follows:

$$
\begin{gathered}
\tilde{\mathbf{P}}_{j}(\omega)=\mathbf{T}\left(\omega, \boldsymbol{\xi}_{\mathbf{N}_{k}}\right) \tilde{\mathbf{F}}_{j}\left(\omega, \boldsymbol{\xi}_{\mathbf{N}_{k}}\right) \\
\mathbf{S}_{\mathbf{P P}}(\omega)=\mathbf{T}\left(\omega, \boldsymbol{\xi}_{\mathbf{N}_{k}}\right) \mathbf{S}_{\mathbf{F F}}\left(\omega, \boldsymbol{\xi}_{\mathbf{N}_{k}}\right) \mathbf{T}\left(\omega, \boldsymbol{\xi}_{\mathbf{N}_{k}}\right)^{H}
\end{gathered}
$$

The midpoint $\xi_{\mathbf{N}_{k}}^{\mathrm{M}}$ and radius $\xi_{\mathbf{N}_{k}}^{\mathrm{R}}$ of $\mathbf{N}_{k}$ can be expressed

$$
\left\{\begin{array}{l}
\xi_{\mathbf{N}_{k}}^{\mathrm{M}}=\frac{\xi_{\mathbf{N}_{k}}^{\mathrm{L}}+\xi_{\mathbf{N}_{k}}^{\mathrm{U}}}{2} \\
\xi_{\mathbf{N}_{k}}^{\mathrm{R}}=\frac{\xi_{\mathbf{N}_{k}}^{\mathrm{U}}-\xi_{\mathbf{N}_{k}}^{\mathrm{L}}}{2}
\end{array}\right.
$$

where $\xi_{\mathbf{N}_{k}}^{\mathrm{L}}$ and $\xi_{\mathbf{N}_{k}}^{\mathrm{U}}$ denote the lower bound and upper bound of $\mathbf{N}_{k}$, respectively.

Theoretically, the extreme value of identified random excitation for any $\mathrm{FE} \mathbf{N}_{k}$ can be solved by two optimization processes

$$
\mathbf{S}_{\mathbf{F F}, \mathbf{N}_{k}}(\omega)=\left[\mathbf{S}_{\mathbf{F F}, \mathbf{N}_{k}}^{\mathbf{L}}(\omega), \mathbf{S}_{\mathbf{F F}, \mathbf{N}_{k}}^{\mathrm{U}}(\omega)\right]=\left[\min _{\xi \in \mathbf{N}_{k}}\left(\mathbf{S}_{\mathbf{F F}}(\omega, \boldsymbol{\xi})\right), \max _{\xi \in \mathbf{N}_{k}}\left(\mathbf{S}_{\mathbf{F F}}(\omega, \xi)\right)\right]
$$

Nevertheless, it is discommodious and time-consuming to identify the extreme value 
of random dynamic loads for all FE by optimization processes. In order to increase computational efficiency, the interval analysis method is used to identify the bounds of random dynamic forces for each FE. According to the first order Taylor series expansion and interval extension, the lower bound $\mathbf{S}_{\mathbf{F F}, \mathbf{N}_{k}}^{\mathbf{L}}(\omega)$ and upper bound $\mathbf{S}_{\mathbf{F F}, \mathbf{N}_{k}}^{\mathrm{U}}(\omega)$ of identified random excitation for each FE $\mathbf{N}_{k}$ can be derived approximately [34].

$$
\left\{\begin{array}{l}
\mathbf{S}_{\mathbf{F F}, \mathbf{N}_{k}}^{\mathbf{L}}(\omega)=\mathbf{S}_{\mathbf{F F}, \mathbf{N}_{k}}\left(\omega, \xi_{\mathbf{N}_{k}}^{\mathrm{M}}\right)-\sum_{l=1}^{q}\left|\frac{\partial \mathbf{S}_{\mathbf{F F}, \mathbf{N}_{k}}\left(\omega, \xi_{\mathbf{N}_{k}}^{\mathrm{M}}\right)}{\partial \xi_{l \mathbf{N}_{k}}}\right| \xi_{l \mathbf{N}_{k}}^{\mathrm{R}} \\
\mathbf{S}_{\mathbf{F F}, \mathbf{N}_{k}}^{\mathrm{U}}(\omega)=\mathbf{S}_{\mathbf{F F}, \mathbf{N}_{k}}\left(\omega, \xi_{\mathbf{N}_{k}}^{\mathrm{M}}\right)+\sum_{l=1}^{q}\left|\frac{\partial \mathbf{S}_{\mathbf{F F}, \mathbf{N}_{k}}\left(\omega, \xi_{\mathbf{N}_{k}}^{\mathrm{M}}\right)}{\partial \xi_{l \mathbf{N}_{k}}}\right| \xi_{l \mathbf{N}_{k}}^{\mathrm{R}}
\end{array}\right.
$$

where $\mathbf{S}_{\mathbf{F F}, \mathbf{N}_{k}}\left(\omega, \xi_{\mathbf{N}_{k}}^{\mathrm{M}}\right)$ denotes the random excitation on the midpoint of FE $\xi_{\mathbf{N}_{k}}^{\mathrm{M}}$, $\frac{\partial \mathbf{S}_{\mathbf{F F}, \mathbf{N}_{k}}\left(\omega, \xi_{\mathbf{N}_{k}}^{\mathrm{M}}\right)}{\partial \xi_{l \mathbf{N}_{k}}}$ represents the sensitivity of random load with respect to the evidence variable $\xi_{l \mathbf{N}_{k}}$.

\subsection{Evidence expression of statistical characteristic of identified random excitation}

The bounds of random excitation on each FE can be obtained by means of the above-mentioned interval analysis method. Meanwhile, utilizing the definition of evidence variable moment and evidence function moment, the bounds of mean value and standard deviation of identified random excitation can be derived to assess the effectiveness and feasibility of the identified results.

\subsubsection{Moment of evidence variable}

Since the FD of evidence variable is analogous to the finite sample space of random variables, the FE can be deemed as the discrete sample subintervals with probabilistic value. For this reason, the moment of random variable is introduced to the evidence theory [33]. 
1) Origin moment

The $c$-th origin moment of evidence variable $\xi$ is defined as follows:

$$
\mathrm{E}\left[\xi^{c}\right]=\sum_{i=1}^{N}\left(\xi_{i}^{\mathbf{I}}\right)^{c} \mathrm{~B}\left(\xi_{i}^{\mathbf{I}}\right)
$$

where $N$ is the number of FE of the evidence variables, $\xi_{i}$ and $\mathrm{B}\left(\xi_{i}^{\mathrm{I}}\right)$ denote the $i$-th $\mathrm{FE}$ and the corresponding BPA, respectively. The first order origin moment $E[\xi]$ represents the mean value $m u_{\xi}$ of the evidence variable $\xi$. When the random parameter is approximated by evidence variable, the random parameter is usually continuous. Thus, the FD of the evidence variable can be expressed as an interval number $\xi^{\mathbf{I}}$, and the FE $\xi_{i}^{\mathbf{I}}$ represents the subinterval of the FD.

2) Central moment

The $c$-th central moment of evidence variable $\xi$ is defined as follows:

$$
\mathrm{E}\left[(\boldsymbol{\xi}-\mathrm{E}[\xi])^{c}\right]=\sum_{i=1}^{N}\left(\xi_{i}^{\mathbf{I}}-\mathrm{E}[\xi]\right)^{c} \mathrm{~B}\left(\xi_{i}^{\mathbf{I}}\right)
$$

Analogously, the second order central moment stands for the variance $\operatorname{var}(\xi)$ of the evidence variable $\xi$.

\subsubsection{Moment of evidence function}

The problem of random load identification for SAC system with evidence variable is essentially a problem of evidence function. Therefore, a method of determining the moment of evidence function is introduced below. Consider the following evidence function

$$
Y=\mathrm{h}(\xi)
$$

The function $h(\xi)$ of evidence variable $\xi$ is also an evidence variable, 
corresponding to an interval and BPA with regard to each FE. In theory, the extreme value of evidence variable function on each FE can be obtained by the following two optimization processes:

$$
Y_{\mathbf{N}_{k}}=\left[Y_{\mathbf{N}_{k}}^{\mathbf{L}}, Y_{\mathbf{N}_{k}}^{\mathrm{U}}\right]=\left[\min _{\xi \in \mathbf{N}_{k}}(Y(\boldsymbol{\xi})), \max _{\boldsymbol{\xi} \in \mathbf{N}_{k}}(Y(\boldsymbol{\xi}))\right]
$$

Moreover, the corresponding BPA of FE $Y_{\mathbf{N}_{k}}$ is equal to the BPA of the FE in joint FD of evidence variables, namely

$$
\mathrm{B}\left(Y_{\mathbf{N}_{k}}\right)=\left\{\begin{array}{cc}
\mathrm{B}\left(\mathbf{N}_{k}\right) & Y=\mathrm{h}(\boldsymbol{\xi}) \\
0 & \text { others }
\end{array}\right.
$$

Calculating the BPA of evidence function and combining the concept of the moment of evidence variables, the moment of evidence function can be described as follows:

1) Mean value

$$
m u_{Y}=\sum_{i=1}^{N} Y_{\mathbf{N}_{k}} \mathrm{~B}\left(Y_{\mathbf{N}_{k}}\right)
$$

2) Variance and standard deviation

$$
\begin{gathered}
\operatorname{var}(Y)=\mathrm{E}\left[\left(Y-m u_{Y}\right)^{2}\right]=\sum_{i=1}^{N}\left(Y_{\mathbf{N}_{k}}-m u_{Y}\right)^{2} \mathrm{~B}\left(Y_{\mathbf{N}_{k}}\right) \\
\operatorname{std}(Y)=\sqrt{\operatorname{var}(Y)}
\end{gathered}
$$

where $m u_{Y}, \operatorname{var}(Y)$ and $\operatorname{std}(Y)$ denote the mean value, variance and standard deviation of evidence function, respectively. By repeated interval analysis of the unknown load on each FE, the BPA of the identified load can be computed by Eq. (20) to Eq. (24) and Eq. (30). In addition, the mean value boundary and variance boundary of the identified random excitation can be obtained based on Eq. (31) and Eq. (32).

The statistical characteristic of identified random excitation obtained by Eq. (31) and 
Eq. (32) is an interval. As the interval would gradually approach the probabilistic result when the number of discrete intervals in the random sample space increases, the statistical characteristics of the identified random load cannot be directly compared with the actual load of the SAC system. Therefore, the nominal mean value $m u_{\text {nom }}\left(\mathbf{S}_{\mathbf{F F}}\right)$ and nominal standard deviation $\operatorname{std}_{\text {nom }}\left(\mathbf{S}_{\mathbf{F F}}\right)$ of identified random excitation for evidence analysis are defined as follows:

$$
\begin{aligned}
& m u_{\text {nom }}\left(\mathbf{S}_{\mathbf{F F}}\right)=\frac{1}{2}\left(m u^{\mathrm{L}}\left(\mathbf{S}_{\mathbf{F F}}\right)+m u^{\mathrm{U}}\left(\mathbf{S}_{\mathbf{F F}}\right)\right) \\
& \operatorname{std}_{\text {nom }}\left(\mathbf{S}_{\mathbf{F F}}\right)=\frac{1}{2}\left(\operatorname{std}^{\mathrm{L}}\left(\mathbf{S}_{\mathbf{F F}}\right)+\operatorname{std}^{\mathrm{U}}\left(\mathbf{S}_{\mathbf{F F}}\right)\right)
\end{aligned}
$$

where the superscript $\mathrm{L}$ and $\mathrm{U}$ represent the lower bound and upper bound of moment of evidence function, respectively. Based on the definition of the nominal mean value and nominal standard deviation, the bounds $\mathrm{S}_{\mathrm{FF}}^{\mathrm{L}}(\omega)$ and $\mathrm{S}_{\mathrm{FF}}^{\mathrm{U}}(\omega)$ of identified random dynamic load are formulated so as to intuitively perceive the effect of the system stochastic parameters on the identifying random excitations as follows:

$$
\left\{\begin{array}{l}
\mathbf{S}_{\mathbf{F F}}^{\mathbf{U}}(\omega)=m u_{\text {nom }}\left(\mathbf{S}_{\mathbf{F F}}\right)+2 \operatorname{std}_{\text {nom }}\left(\mathbf{S}_{\mathbf{F F}}\right) \\
\mathbf{S}_{\mathbf{F F}}^{\mathbf{L}}(\omega)=m u_{\text {nom }}\left(\mathbf{S}_{\mathbf{F F}}\right)-2 \operatorname{std}_{\text {nom }}\left(\mathbf{S}_{\mathbf{F F}}\right)
\end{array}\right.
$$

\section{Improved regularization method for random excitation identification based on sound pressure responses}

In practical engineering circumstances, not only the obtained FRF $\mathrm{T}(\omega)$, which stands for the dynamic characteristics of the coupled system, but also the measured acoustic pressure responses contain error inevitably. With regard to the FRF containing error, the evidence theory is applied to solve the problem of SAC system with random parameters in the above section. With respect to the acoustic pressure responses containing noises, it has a 
great influence on the error propagation and amplification of identifying random excitation, which results in the ill-posed problem. Therefore, the error of random dynamic loads to be identified is analyzed in this section. Subsequently, an improved regularization method is developed to handle the ill-posedness.

\subsection{Error analysis}

Considering the noise contained in the measuring acoustic pressure responses, the PSD of dynamic responses in the acoustic cavity can be reformulated as

$$
\mathbf{S}_{\mathbf{P P}}^{\boldsymbol{\delta}}(\omega)=\mathbf{S}_{\mathbf{P P}}(\omega)+\Delta \mathbf{S}_{\mathbf{P P}}(\omega)=\mathbf{T}(\omega) \mathbf{S}_{\mathbf{F F}}(\omega) \mathbf{T}(\omega)^{H}+\Delta \mathbf{S}_{\mathbf{P P}}(\omega)
$$

where $\mathrm{S}_{\mathrm{PP}}^{\delta}$ denotes the PSD matrix of the measured acoustic pressure responses, $\mathrm{S}_{\mathrm{PP}}$ and $\Delta \mathrm{S}_{\mathrm{PP}}$ represent the PSD matrices of real acoustic pressure responses and measurement error respectively.

Hence, the relation between identifying random dynamic loads and measured acoustic pressure responses can be rewritten as

$$
\mathbf{S}_{\mathbf{P P}}^{\boldsymbol{\delta}}(\omega)=\mathbf{T}(\omega) \mathbf{S}_{\mathbf{F F}}^{\boldsymbol{\delta}}(\omega) \mathbf{T}(\omega)^{H}
$$

in which $\mathbf{S}_{\mathbf{F F}}^{\boldsymbol{\delta}}(\omega)=\mathbf{S}_{\mathbf{F F}}(\omega)+\Delta \mathbf{S}_{\mathbf{F F}}(\omega), \mathbf{S}_{\mathbf{F F}}(\omega)$ denotes the PSD matrix of actual random dynamic loads, $\Delta \mathbf{S}_{\mathbf{F F}}(\omega)$ is known as the error matrix of the random excitation to be identified.

The inverse process of determining the random excitation is an ill-posed problem resulting from the measurement error of acoustic pressure and ill-conditioned FRF matrix. Thus, it is the primary task to study the affecting factors on relative error of identified random loads.

From Eq. (37), the corresponding equation Eq. (13) with error terms can be 
reformulated as

$$
\tilde{\mathbf{P}}_{j}^{\delta}(\omega)=\mathbf{T}(\omega) \tilde{\mathbf{F}}_{j}^{\delta}(\omega)
$$

where $\quad \tilde{\mathbf{P}}_{j}^{\delta}(\omega)=\tilde{\mathbf{P}}_{j}(\omega)+\Delta \tilde{\mathbf{P}}_{j}(\omega) \quad, \quad \tilde{\mathbf{F}}_{j}(\omega)=\tilde{\mathbf{F}}_{j}(\omega)+\Delta \tilde{\mathbf{F}}_{j}(\omega), \Delta \tilde{\mathbf{P}}_{j}(\omega)$ and $\Delta \tilde{\mathbf{F}}_{j}(\omega)$ denote the error terms of the pseudo acoustic pressure responses and pseudo excitation, respectively. And the pseudo responses vector is decomposed by the PSD matrix of measured acoustic pressure responses as follows:

$$
\mathbf{S}_{\mathbf{P P}}^{\boldsymbol{\delta}}(\omega)=\sum_{j=1}^{r} \tilde{\mathbf{P}}_{j}^{\delta}(\omega) \tilde{\mathbf{P}}_{j}^{\delta}(\omega)^{H}=\sum_{j=1}^{r} \beta_{j} \boldsymbol{\Phi}_{j} \boldsymbol{\Phi}_{j}{ }^{H} \quad(j=1,2, \ldots, r)
$$

Accordingly, the relative error of pseudo excitations identification can be derived (see Appendix A)

$$
\frac{\left\|\Delta \tilde{\mathbf{F}}_{j}(\omega)\right\|}{\left\|\tilde{\mathbf{F}}_{j}(\omega)\right\|} \leq\left\|\mathbf{T}(\omega)^{+}\right\|\|\mathbf{T}(\omega)\| \frac{\left\|\Delta \tilde{\mathbf{P}}_{j}(\omega)\right\|}{\left\|\tilde{\mathbf{P}}_{j}(\omega)\right\|}
$$

in which $\|\cdot\|$ represents the matrix norm, the superscript + is known as the generalized inversion.

According to the condition number theory, Eq. (40) can be expressed as

$$
\frac{\left\|\Delta \tilde{\mathbf{F}}_{j}(\omega)\right\|}{\left\|\tilde{\mathbf{F}}_{j}(\omega)\right\|} \leq \operatorname{cond}(\mathbf{T}(\omega)) \frac{\left\|\Delta \tilde{\mathbf{P}}_{j}(\omega)\right\|}{\left\|\tilde{\mathbf{P}}_{j}(\omega)\right\|}
$$

where $\operatorname{cond}(\mathbf{T}(\omega))=\left\|\mathbf{T}(\omega)^{+}\right\|\|\mathbf{T}(\omega)\|$.

It can be seen from Eq. (41) that the measurement error in acoustic pressure responses $\frac{\left\|\Delta \tilde{\mathbf{P}}_{j}(\omega)\right\|}{\left\|\tilde{\mathbf{P}}_{j}(\omega)\right\|}$ and the condition number $\operatorname{cond}(\mathbf{T}(\omega))$ are the determinant factors on the upper bounds of relative error of identified pseudo excitations $\frac{\left\|\Delta \tilde{\mathbf{F}}_{j}(\omega)\right\|}{\left\|\tilde{\mathbf{F}}_{j}(\omega)\right\|}$. Therefore, in order to 
mitigate $\frac{\left\|\Delta \tilde{\mathbf{F}}_{j}(\omega)\right\|}{\left\|\tilde{\mathbf{F}}_{j}(\omega)\right\|}$, the composite condition number method [49] is applied by means of decreasing the condition number. Moreover, owing to the effect of $\operatorname{cond}(\mathbf{T}(\omega))$ on identification error, an improved regularization method is proposed to handle the ill-posed problem in next section.

\subsection{Improved regularization method}

With regard to the load identification problem described in Eq. (42), the small singular values of $\mathbf{T}(\omega)$ are basically responsible for the ill-posed problems. The smaller the singular value is, the more serious the measuring noise in acoustic pressure responses is amplified. The singular values tending to zero result in the instability and inaccuracy of identifying forces. Thus, to obtain accurate and available solutions, it is quite necessary to not only reduce the impact of small singular value but also retain sufficient information of the identifying loads, which is the purpose of regularization method.

$$
\tilde{\mathbf{P}}_{j}(\omega)=\mathbf{T}(\omega) \tilde{\mathbf{F}}_{j}(\omega)
$$

Converting Eq. (42) into a linear least-squares problem as follows

$$
\min _{\widetilde{\mathbf{F}}_{j}}\left\|\mathbf{T}(\omega) \widetilde{\mathbf{F}}_{j}(\omega)-\widetilde{\mathbf{P}}_{j}(\omega)\right\|
$$

According to the theorem of Tikhonov regularization method [50], the minimization problem in Eq. (43) is replaced by a penalized least-squares problem described in Eq. (44) leading to mitigate the ill-posed problem.

$$
\tilde{\mathbf{F}}_{j}^{\varepsilon}(\omega)=\arg \min \left\{\mid \mathbf{T}(\omega) \widetilde{\mathbf{F}}_{j}(\omega)-\widetilde{\mathbf{P}}_{j}(\omega)\left\|^{2}+\varepsilon^{2}\right\| \widetilde{\mathbf{F}}_{j}(\omega) \|^{2}\right\}
$$

where $\varepsilon$ represents the regularization parameter, and the regularized solution $\tilde{\mathbf{F}}_{j}^{\varepsilon}(\omega)$ can be determined by the least squares problem in the following form: 


$$
\min _{\widetilde{\mathbf{F}}_{j}(\omega)}\left\|\left(\begin{array}{c}
\mathbf{T}(\omega) \\
\varepsilon \mathbf{I}
\end{array}\right) \tilde{\mathbf{F}}_{j}(\omega)-\left(\begin{array}{c}
\tilde{\mathbf{P}}_{j}(\omega) \\
\mathbf{0}
\end{array}\right)\right\|
$$

Eq. (45) can be reformulated as

$$
\left(\mathbf{T}(\omega)^{H} \mathbf{T}(\omega)+\varepsilon^{2} \mathbf{I}\right) \tilde{\mathbf{F}}_{j}(\omega)=\mathbf{T}(\omega)^{H} \widetilde{\mathbf{P}}_{j}(\omega)
$$

On the basis of singular value decomposition (SVD) theory [51], the FRF matrix $\mathbf{T}(\omega)$ can be decomposed as

$$
\mathbf{T}(\omega)=\mathbf{U d i a g}\left(\boldsymbol{\theta}_{n}\right) \mathbf{V}^{H}=\sum_{i=1}^{n} \mathbf{u}_{i} \theta_{i} \mathbf{v}_{i}^{H}
$$

where $\mathbf{U}=\left(\mathbf{u}_{1}, \mathbf{u}_{2}, \cdots, \mathbf{u}_{m}\right), \mathbf{V}=\left(\mathbf{v}_{1}, \mathbf{v}_{2}, \cdots, \mathbf{v}_{n}\right), \mathbf{u}_{i}$ represents the left singular vector of FRF matrix $\mathbf{T}(\omega)$ and $\mathbf{v}_{i}$ denotes the right singular vector of $\mathbf{T}(\omega)$; $\theta_{1} \geq \theta_{2} \geq \cdots \geq \theta_{n} \geq 0$ stand for the singular value of $\mathbf{T}(\omega)$.

Combining Eq. (46) with Eq. (47), the identifying pseudo excitation can be reformulated as follows:

$$
\tilde{\mathbf{F}}_{j}(\omega)=\operatorname{Vdiag}\left(g\left(\varepsilon, \theta_{i}\right) \theta_{i}^{-1}\right) \mathbf{U}^{H} \tilde{\mathbf{P}}_{j}(\omega)=\sum_{i=1}^{n} g\left(\varepsilon, \theta_{i}\right) \theta_{i}^{-1}\left(\mathbf{u}_{i}^{H} \tilde{\mathbf{P}}_{j}(\omega)\right) \mathbf{v}_{i}
$$

in which $g\left(\varepsilon, \theta_{i}\right)$ is the regularization operator, and the Tikhonov regularization operator is expressed as

$$
g\left(\varepsilon, \theta_{i}\right)=\frac{\theta_{i}^{2}}{\varepsilon^{2}+\theta_{i}^{2}}
$$

It is noted that the regularization method adjusts the weight between minimization of the residual norm and minimization of solution norm according to the regularization parameter. Moreover, the solution norm $\left\|\widetilde{\mathbf{F}}_{j}(\omega)\right\|$ is a monotone non-increasing function of regularization parameter $\varepsilon$, on the contrary, the residual norm $\left\|\mathbf{T}(\omega) \tilde{\mathbf{F}}_{j}(\omega)-\widetilde{\mathbf{P}}_{j}(\omega)\right\|$ is a monotone non-decreasing function of $\varepsilon$. Obviously, a large regularization parameter $\varepsilon$ 
results in a small solution norm at the cost of a large residual norm, which guarantees the approximation between evaluating acoustic pressure and the measuring one. However, a small regularization parameter $\varepsilon$ has the opposite impact leading to achieve more stable identified solutions. In addition, we can infer that the sensitivity of the $\tilde{\mathbf{F}}_{j}^{\varepsilon}(\omega)$ with respect to perturbations in $\mathbf{T}(\omega)$ and $\tilde{\mathbf{P}}_{j}(\omega)$ is controlled by $\varepsilon$. Therefore, it is a fundamental problem of regularization method to put forward an appropriate parameter $\varepsilon$ for balancing the stability and accuracy of the regularized solutions.

At present, the generalized cross-validation (GCV) criterion is widely applied to single out the optimal regularization parameter. The GCV function can be expressed as follows:

$$
\mathrm{C}(\varepsilon)=\frac{\left\|\mathbf{T}(\omega) \tilde{\mathbf{F}}_{j}(\omega)-\tilde{\mathbf{P}}_{j}(\omega)\right\|^{2}}{\left(\operatorname{TRA}\left[\mathbf{I}-\mathbf{T}(\omega)\left(\mathbf{T}(\omega)^{H} \mathbf{T}(\omega)+\varepsilon^{2} \mathbf{I}\right)^{-1} \mathbf{T}(\omega)^{H}\right]\right)^{2}}
$$

where TRA[.] represents the matrix trace. The optimal regularization parameter is given by computing the minimal point of $\mathrm{C}(\varepsilon)$.

Nevertheless, the most of the applications of regularization methods concentrate in load identification in time domain and deterministic load identification in frequency domain. Considering the particularity of random excitation identification and SAC system, a novel improved regularization method is developed to overcome the ill-posed problems in this section. According to the theory of SVD and characteristic of pseudo response, with the increase of order, the eigenvalue $\beta_{j}$ decreases and the corresponding pseudo responses vector contains less information of measured acoustic pressure responses PSD matrix. Moreover, in view of the impact of $\varepsilon$ on the solution norm and residual norm, for the fore 
orders of pseudo responses, which contain the most of the information of measured acoustic pressure responses, the small regularization parameter should be applied to preserve more information and ensure the accuracy of identified random excitation. However, with respect to the latter orders of pseudo responses, which involve less information, the large regularization parameter should be adopted to guarantee the stability of identified random excitation. In addition, combining the influence of condition number cond $(\mathrm{T}(\omega))$ on identification error, a novel improved regularization parameter is developed as follows:

$$
\hat{\varepsilon}_{j}=\frac{\operatorname{cond}(\mathbf{T}(\omega)) \sqrt{\beta_{1}} \varepsilon}{\operatorname{cond}(\mathbf{T}(\omega))_{\max } \sqrt{\beta_{j}}}
$$

where $\hat{\varepsilon}_{j}$ represents the improved regularization parameter for $j$-th order of pseudo acoustic pressure, $\beta_{j}$ denotes the eigenvalue of measured acoustic pressure PSD matrix, $\varepsilon$ is determined by GCV criterion, and $\operatorname{cond}(\mathbf{T}(\omega))_{\max }$ stands for the peak value of condition number of FRF matrix $\mathbf{T}(\omega)$ in the whole frequency domain.

Substituting the improved regularization parameter into Eq. (48), the identifying pseudo excitation vector can be obtained

$$
\tilde{\mathbf{F}}_{j}(\omega)=\operatorname{Vdiag}\left(g\left(\hat{\varepsilon}_{j}, \theta_{i}\right) \theta_{i}^{-1}\right) \mathbf{U}^{H} \tilde{\mathbf{P}}_{j}(\omega)=\sum_{i=1}^{n} g\left(\hat{\varepsilon}_{j}, \theta_{i}\right) \theta_{i}^{-1}\left(\mathbf{u}_{i}^{H} \widetilde{\mathbf{P}}_{j}(\omega)\right) \mathbf{v}_{i}
$$

\section{Numerical Examples}

In order to effectively verify the proposed methods in the present study, a simple plate coupled with the cuboid cavity is firstly investigated to clarify how to identify random excitation based on the sound pressure responses. Through calculating the bounds of random excitation on each FE using the improved regularization method and interval analysis, the 
bounds of random excitation are obtained by evidence method and compared with those by matrix perturbation method. Then, in comparison with MPI method and Tikhonov regularization method, the proposed improved regularization method applied to a vehicle liftgate coupled with the interior cavity, is validated to be more efficient and superior.

\subsection{Procedure of random excitation identification}

On the basis of evidence theory and improved regularization method, the flow of random dynamic loads identification for stochastic SAC system is expressed as the following steps:

(1) Determine the random acoustic pressure responses $S_{P P}(\omega)$ using the given constructed random dynamic loads and finite element analysis, and then add a random noise to the computational acoustic pressure responses PSD matrix, and decompose the polluted responses PSD matrix $\mathrm{S}_{\mathrm{PP}}^{\delta}(\omega)$ into several pseudo response vectors $\mathbf{P}_{j}(\omega)$.

(2) Decompose the FRF matrix $\mathrm{T}(\omega)$ by SVD, and compute the improved regularization parameter $\hat{\varepsilon}_{j}$ by Eq. (51).

(3) Discretize the PDF of random parameters $\kappa$ with several subintervals, calculate the bounds of random excitation on each FE using the improved regularization method and interval analysis.

(4) Calculate the mean value and standard deviation of the unknown random dynamic loads based on the evidence theory.

(5) Compute the upper bound $\mathbf{S}_{\mathbf{F F}}^{\mathbf{U}}(\omega)$ and lower bound $\mathbf{S}_{\mathbf{F F}}^{\mathbf{L}}(\omega)$ of random dynamic forces based on the definition of the nominal mean value and nominal 
standard deviation.

\subsection{A simple plate coupled with the cuboid cavity}

To demonstrate the superiority of evidence theory for the SAC system involving random parameter with large coefficient of variation and multimodal distribution, a verification based on simulation is studied on a simple plate coupled with the cuboid cavity. Meanwhile, the effectiveness and applicability of the novel sound pressure-based algorithm and the proposed improved regularization method is verified in the numerical example. As outlined in Fig. 3, the size of the plate and the cuboid acoustic cavity are set as $768 \times 368 \times 8 \mathrm{~mm}$ and $768 \times 368 \times 368 \mathrm{~mm}$, respectively. The properties of the structural materials include: the Young's modulus $E=71 \mathrm{GPa}$, the material density $\rho=2700 \mathrm{~kg} / \mathrm{m}^{3}$, the Poisson's ratio $v=0.31$, and the structural damping factor $\mu=0.06$. The properties of the cuboid acoustic cavity are set as follows: the sound velocity $v_{a}=340 \mathrm{~m} / \mathrm{s}$ and the mass density $\rho_{a}=1.2 \mathrm{~kg} / \mathrm{m}^{3}$. The four sides of the plate are fully constrained, and the top surface of the cuboid acoustic cavity is coupled with the vibration of the structural plate.

Three exciting positions on the structural plate are singled out in $z$-direction at random, and ten candidates for acoustic pressure points in the acoustic cavity are selected randomly. In order to reduce the error amplification owing to the big condition number of $\mathbf{T}(\omega)$, the composite condition number method is employed to sort out five measurement locations as the best combination from ten ones. Consequently, the best and worst groups of measurement points are obtained by the comparison of the composite condition number of all optional combinations as follows: the best combination 1,3,5,8,9 and the worst combination 2,6,8,9,10. It can be observed from Fig. 4 that the best group is able to mitigate 
the condition number markedly.

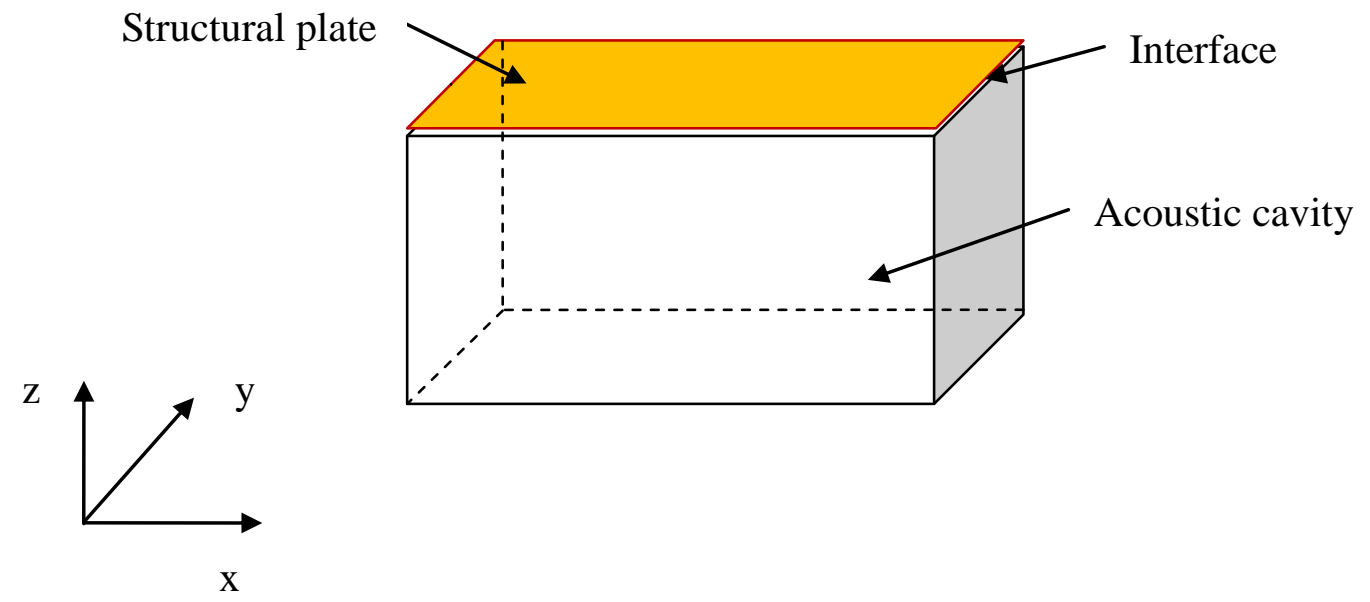

Fig. 3. The model of simple plate and the coupled cuboid cavity.

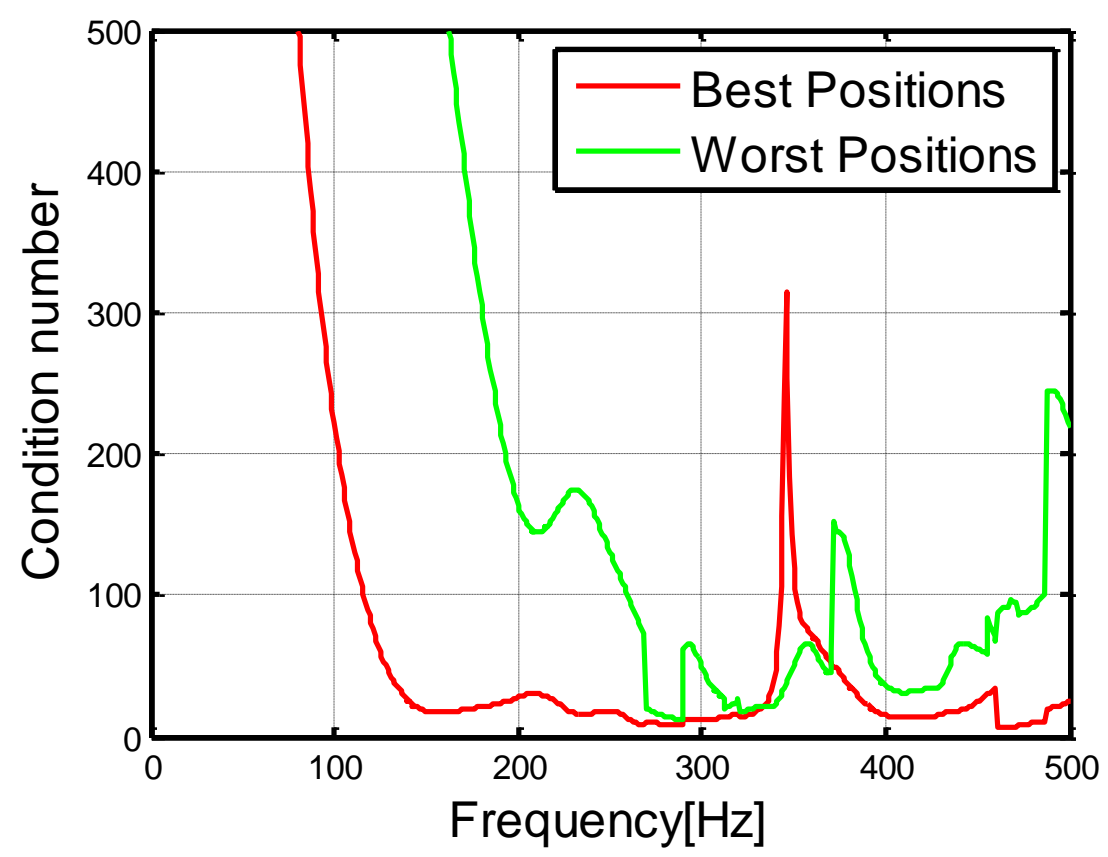

Fig. 4. $\operatorname{cond}(\mathbf{T}(\omega))$ of the best group of measurement positions and worst one.

Considering the random parameters with large coefficient of variation and multimodal distribution, we assume that the elastic modulus of the structural plate is a random parameter $\kappa$ following the bimodal PDF defined by Eq. (53), whose mean value and standard deviation shown in Fig. 5 are $m u=0.703 \times 10^{11}, \operatorname{std}(\kappa)=6.68 \times 10^{8}$. 


$$
w(x)=a \frac{1}{\sqrt{2 \pi} \tau_{1}} \exp \left(-\frac{\left(x-\mu_{1}\right)^{2}}{2 \tau_{1}^{2}}\right)+(1-a) \frac{1}{\sqrt{2 \pi} \tau_{2}} \exp \left(-\frac{\left(x-\mu_{2}\right)^{2}}{2 \tau_{2}^{2}}\right)
$$

where $\mu_{1}=0.7 \times 10^{11}, \tau_{1}=1.6 \times 10^{8}, \mu_{2}=0.71 \times 10^{11}, \tau_{2}=2.1 \times 10^{8}, a=0.2$.

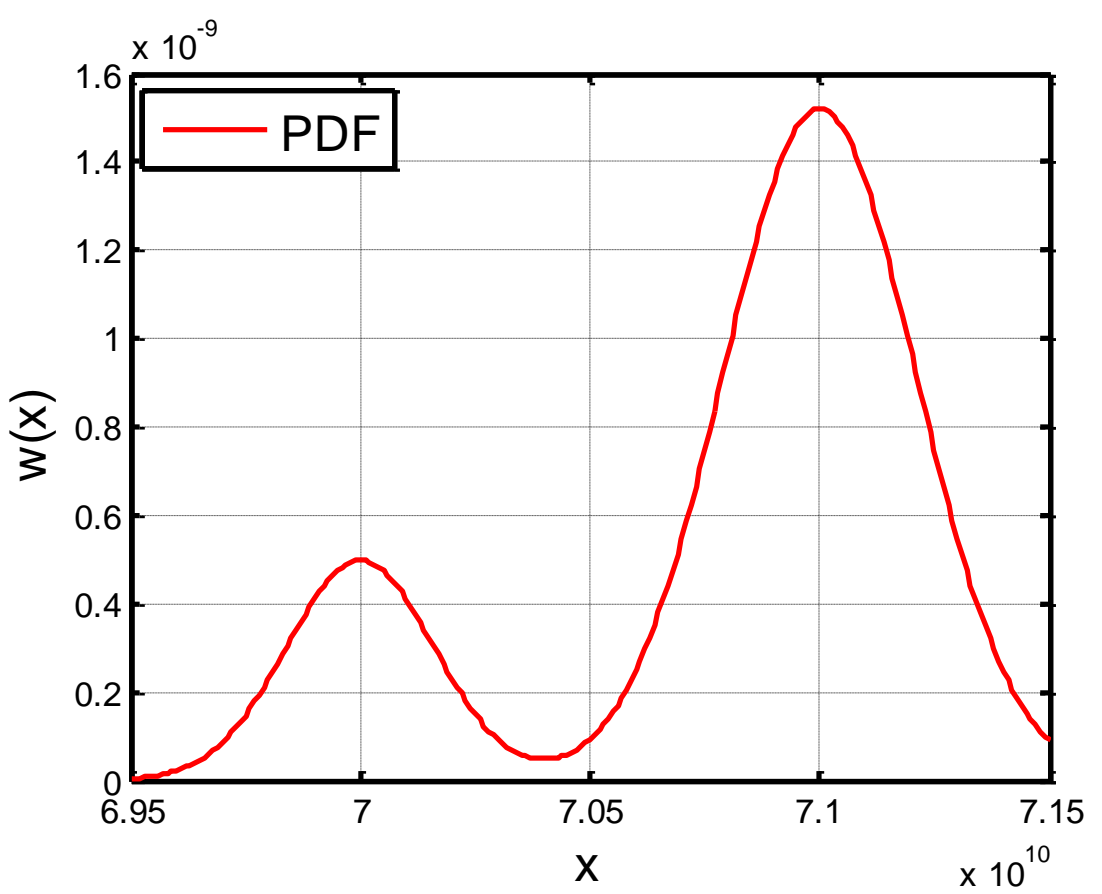

Fig. 5. The bimodal PDF of the random parameter $\kappa$.

Selecting a random value of $\kappa$, the random dynamic acoustic pressure responses are computed by finite element simulation on the basis of the pre-given PSD matrix of random dynamic loads on the structural plate. Additionally, as shown in Fig. 6, a random noise is added to the computational acoustic pressure so as to simulate the actual measured responses. 


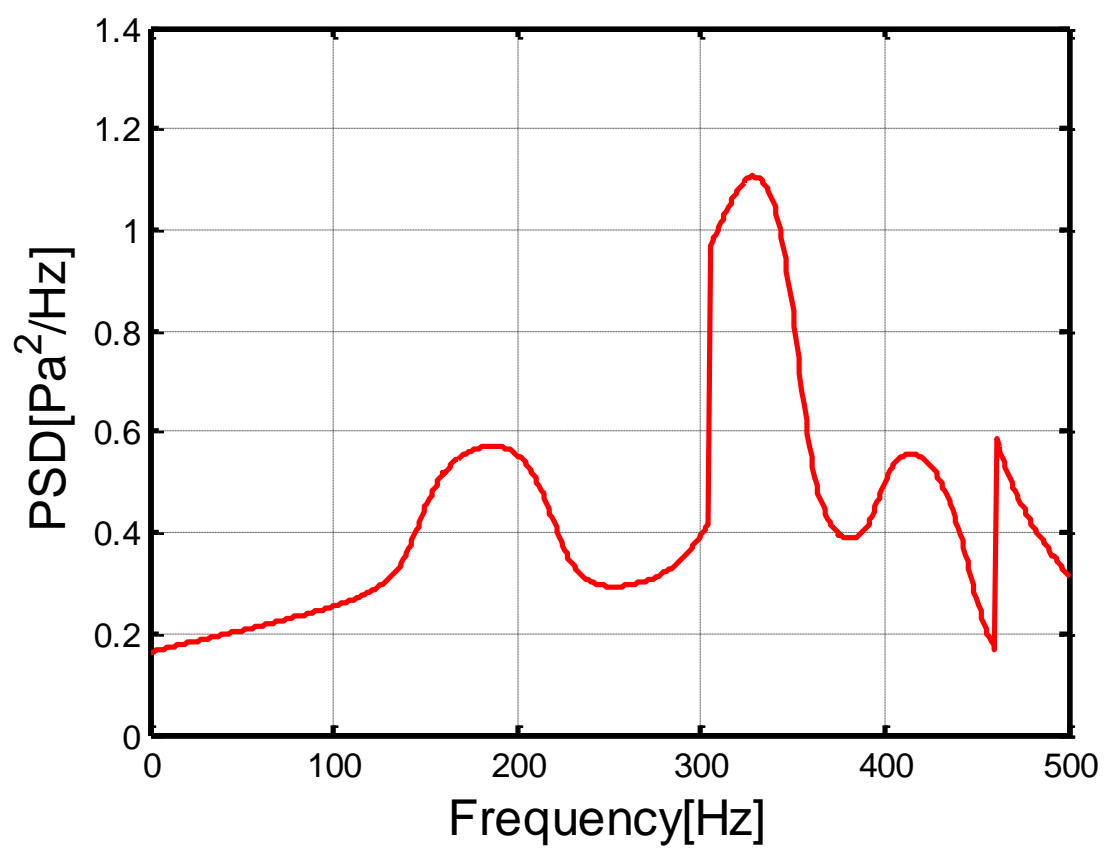

Fig. 6. Autopower spectral density (Auto-PSD) of the random acoustic pressure of response position 1 with $5 \%$ random noise.

Discretizing the PDF of random parameters $\kappa$ with two subintervals, the stochastic SAC system problem is converted to the evidence analysis problem. The bounds of random dynamic loads on each FE are obtained using the improved regularization method and interval analysis. In the light of the theory described in Section 3.2, the mean value and standard deviation of the identifying random excitation are derived by the means of evidence analysis. Moreover, based on the matrix perturbation method, the mean value and standard deviation of the unknown random excitation are also computed so as to compare with the result of evidence analysis. It is noted from the results comparison that both matrix perturbation and evidence analysis can achieve the mean value of identified random excitation with higher precision as shown in Fig. 7. Nevertheless, aimed at the high order moments, such as the standard deviation, the results identified by evidence theory method are more accurate compared with the results by matrix perturbation mehod as shown in Fig. 
8. EM described in figures denotes the evidence moment.

However, even the best combination is selected, the condition numbers are still too high in the low and high frequencies. This is also the reason why the identified mean of the dynamic load has a larger fluctuation in the low and high frequencies than in the intermediate. The identified standard deviation has a larger fluctuation only in the high frequency since the identified random excitation value is smaller in the low frequency.

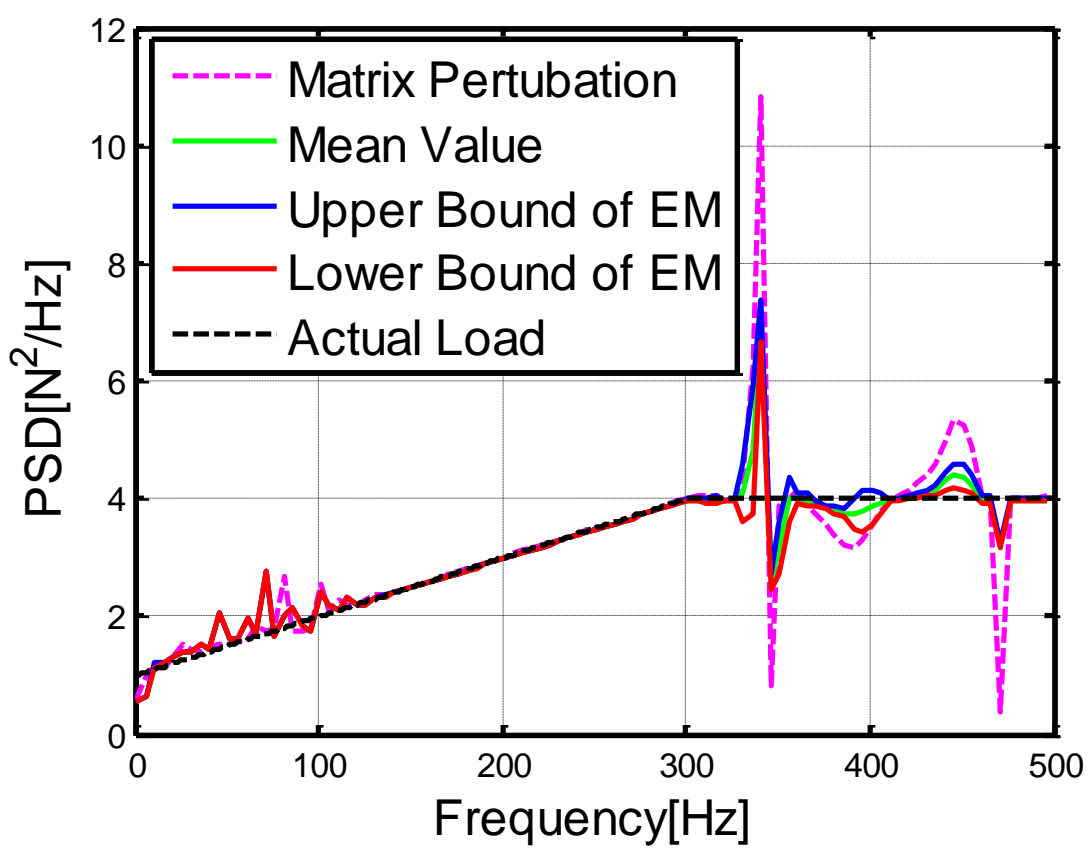

(a) 


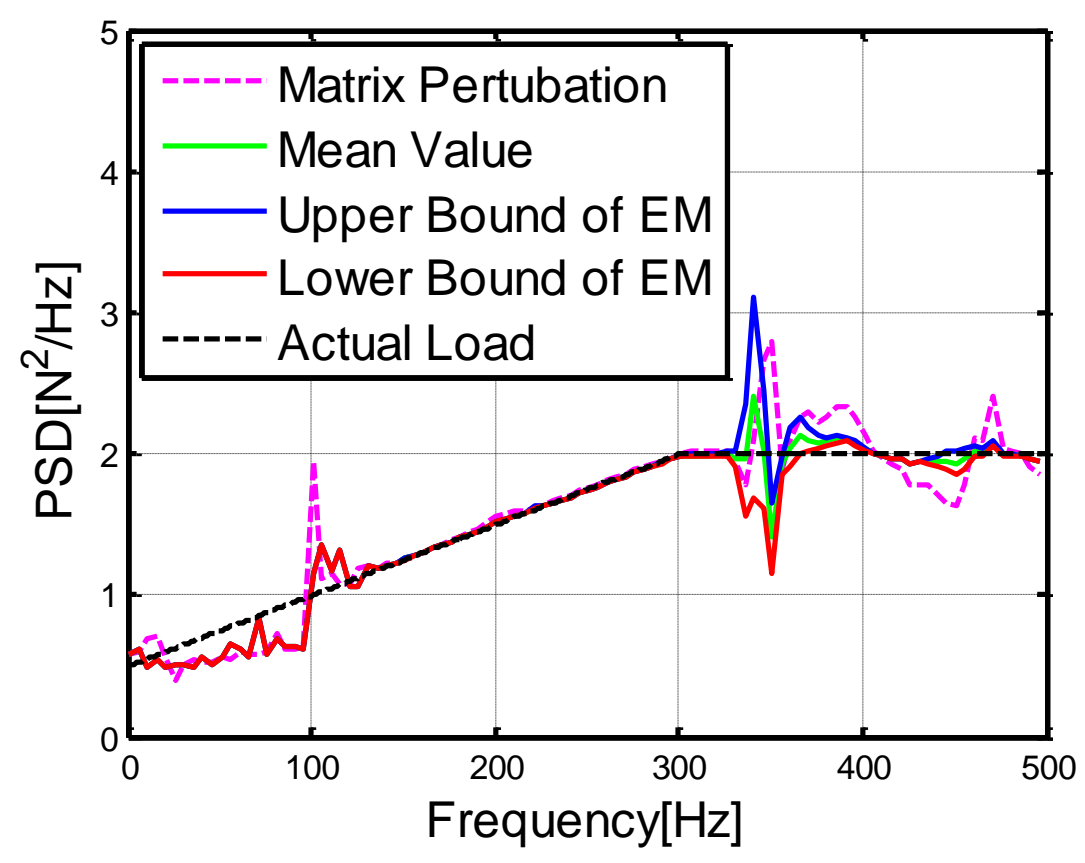

(b)

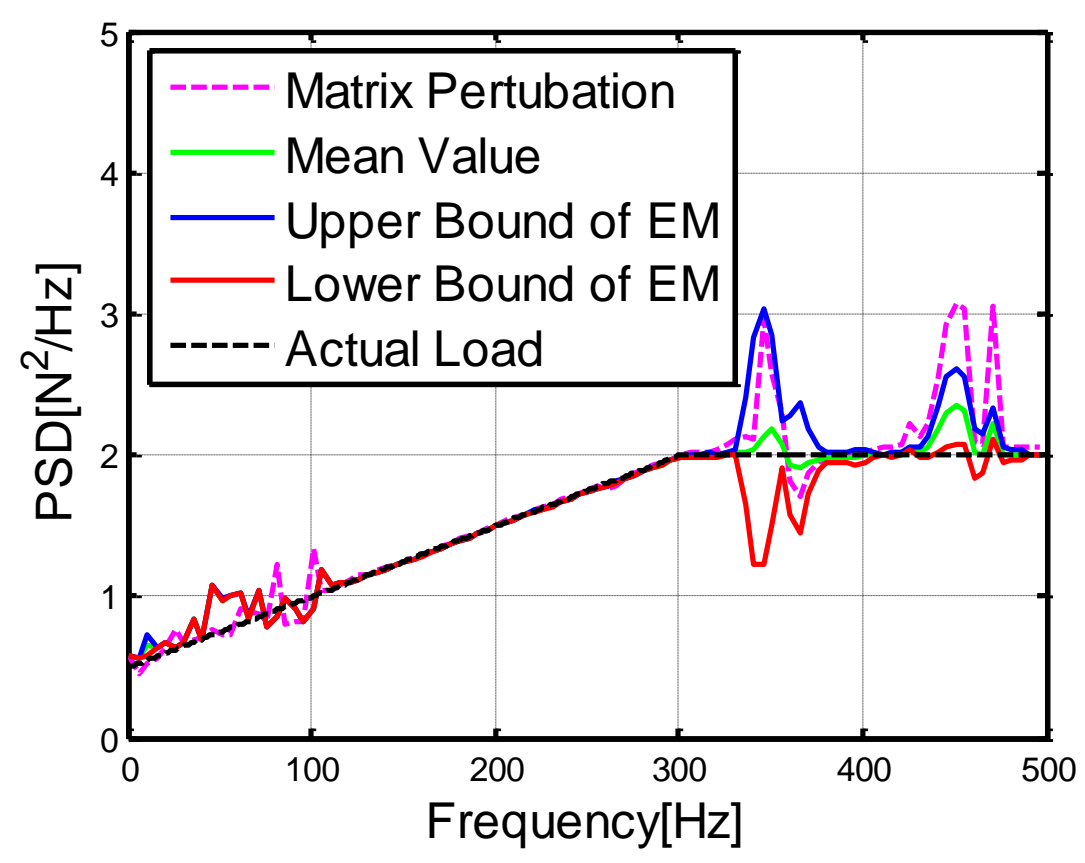

(c)

Fig. 7. The Auto-PSD mean value of random loads computed by matrix perturbation method and evidence theory: (a) exciting position I ; (b) exciting position II ; (c) exciting position III. 


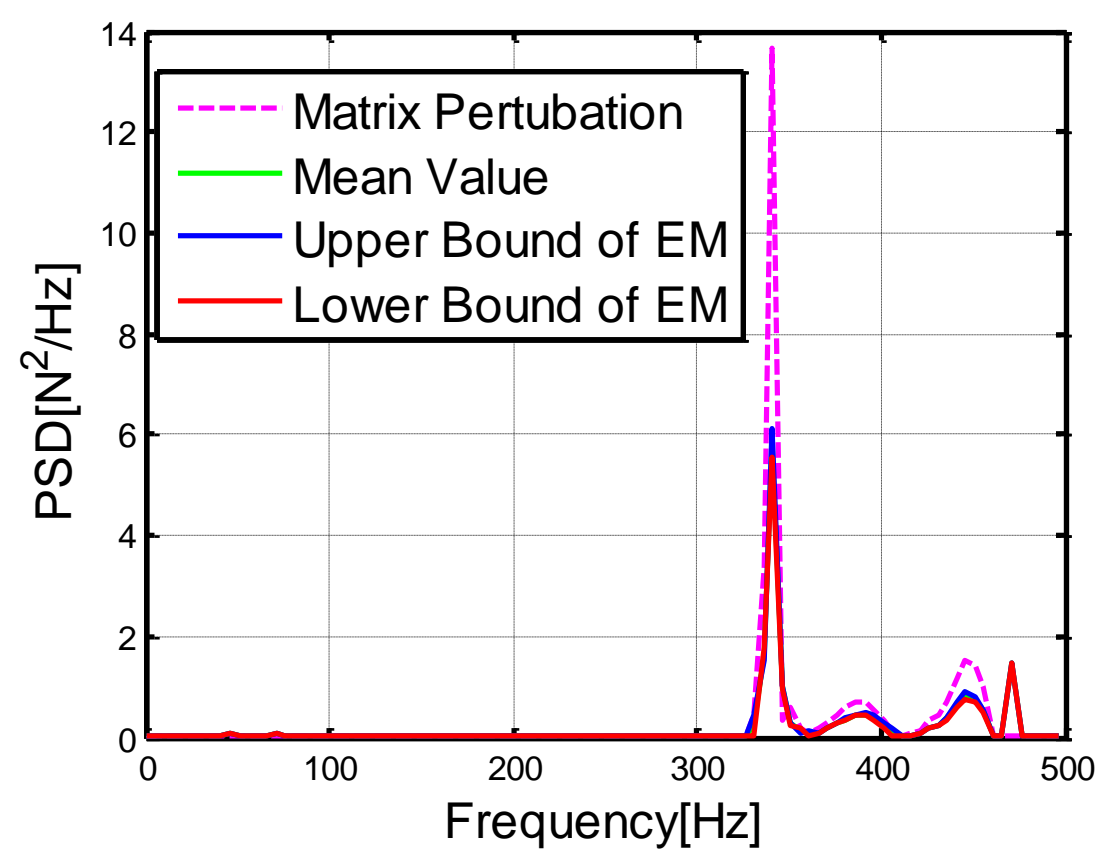

(a)

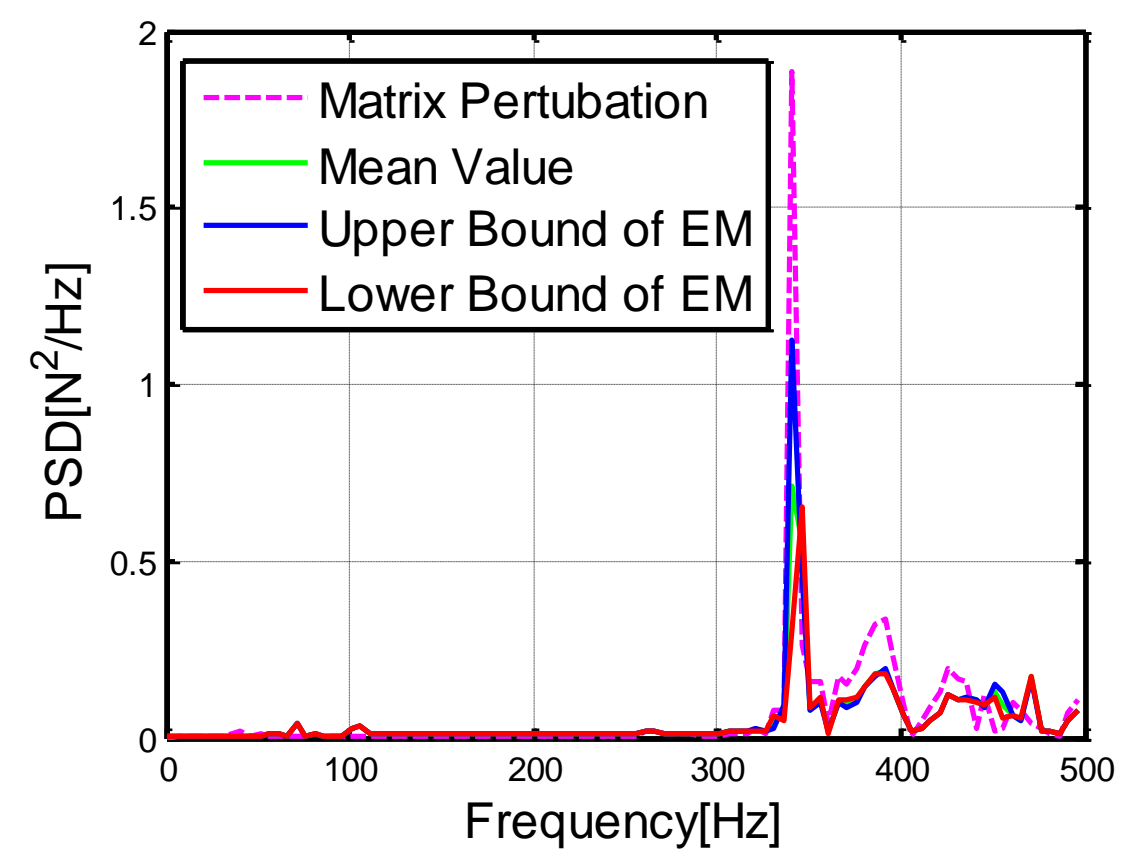

(b) 


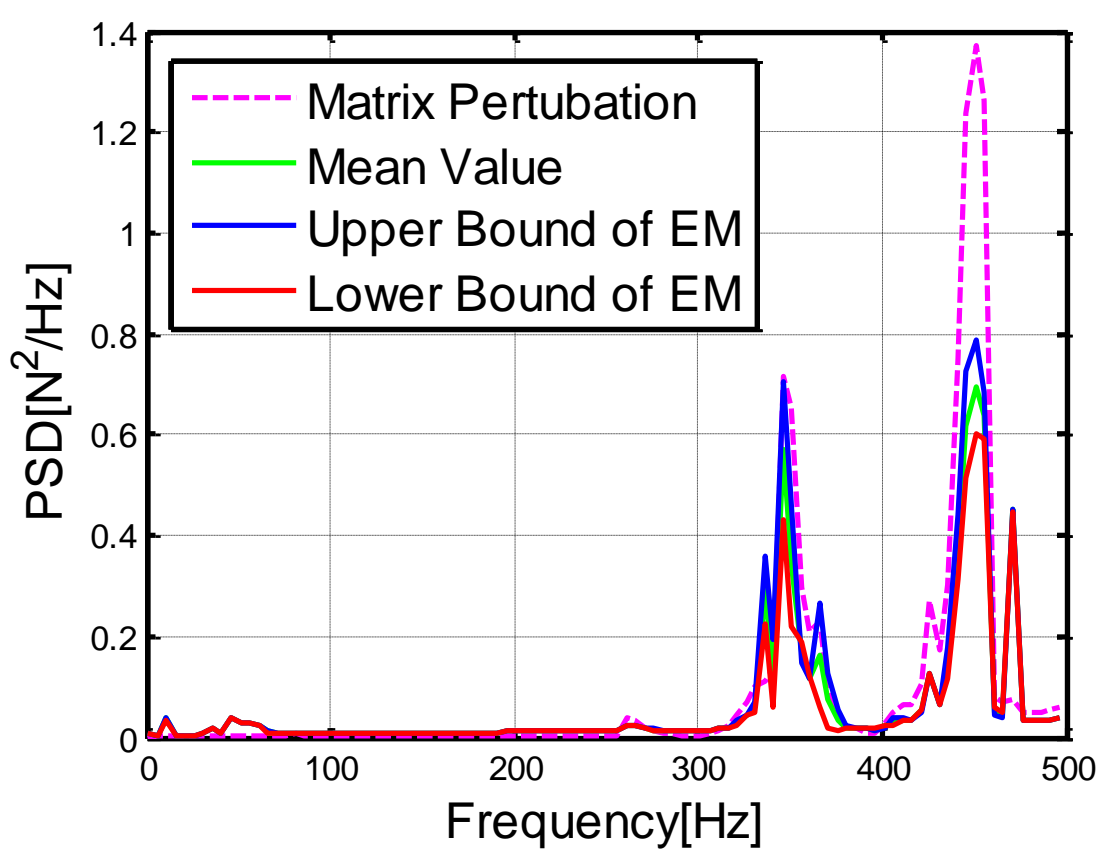

(c)

Fig. 8. The Auto-PSD standard deviation of random loads computed by matrix perturbation method and evidence theory: (a) exciting position I ; (b) exciting position II ; (c) exciting position III.

Additionally, to intuitively perceive the impact of the structural stochastic parameters on the identifying random dynamic loads, the statistical characteristics of the unknown random excitation, namely the bounds $\mathrm{S}_{\mathrm{FF}}^{\mathrm{L}}(\omega)$ and $\mathrm{S}_{\mathrm{FF}}^{\mathrm{U}}(\omega)$ shown in Fig. 9 and Fig. 10 are calculated based on the evidence analysis theory and matrix perturbation method, respectively. It can be seen that the bounds obtained by evidence analysis mostly bracket the actual loads, and the results from evidence analysis are more accurate than those by perturbation method.

To conclude, through the random excitation identification study on the thin plate coupled with the cuboid cavity, the novel sound pressure-based method and the improved regularization method are proved to be effective and applicative in practical engineering circumstance. Moreover, with respect to the SAC system involving random parameter with 
large coefficient of variation and multimodal distribution, the proposed method based on evidence theory is able to identify the unknown random excitation more effectively and accurately compared with the matrix perturbation method.

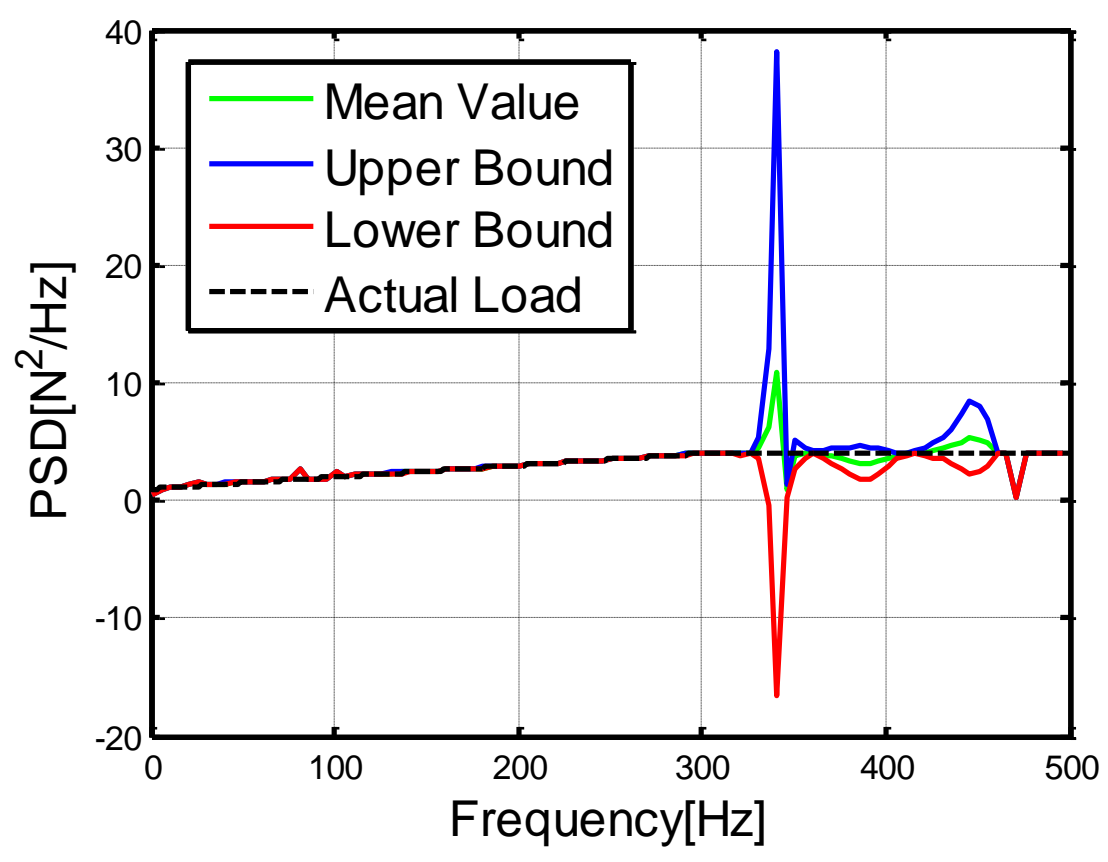

(a)

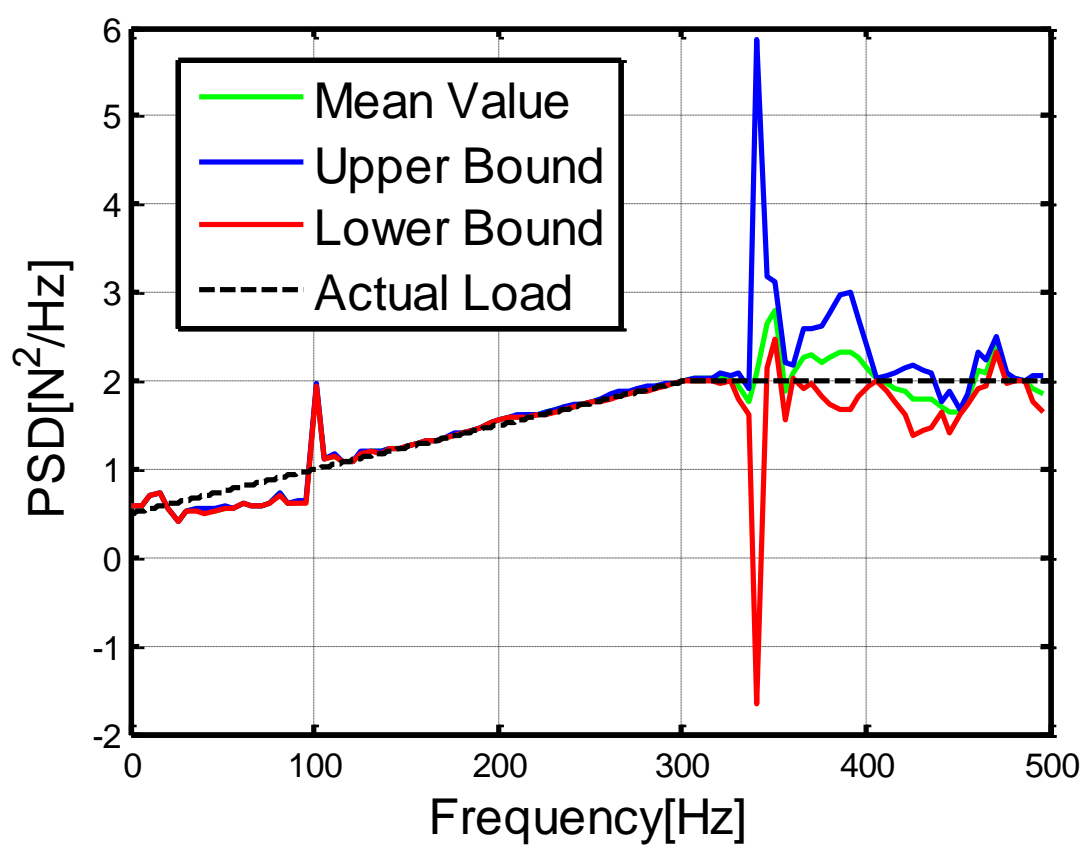


(b)

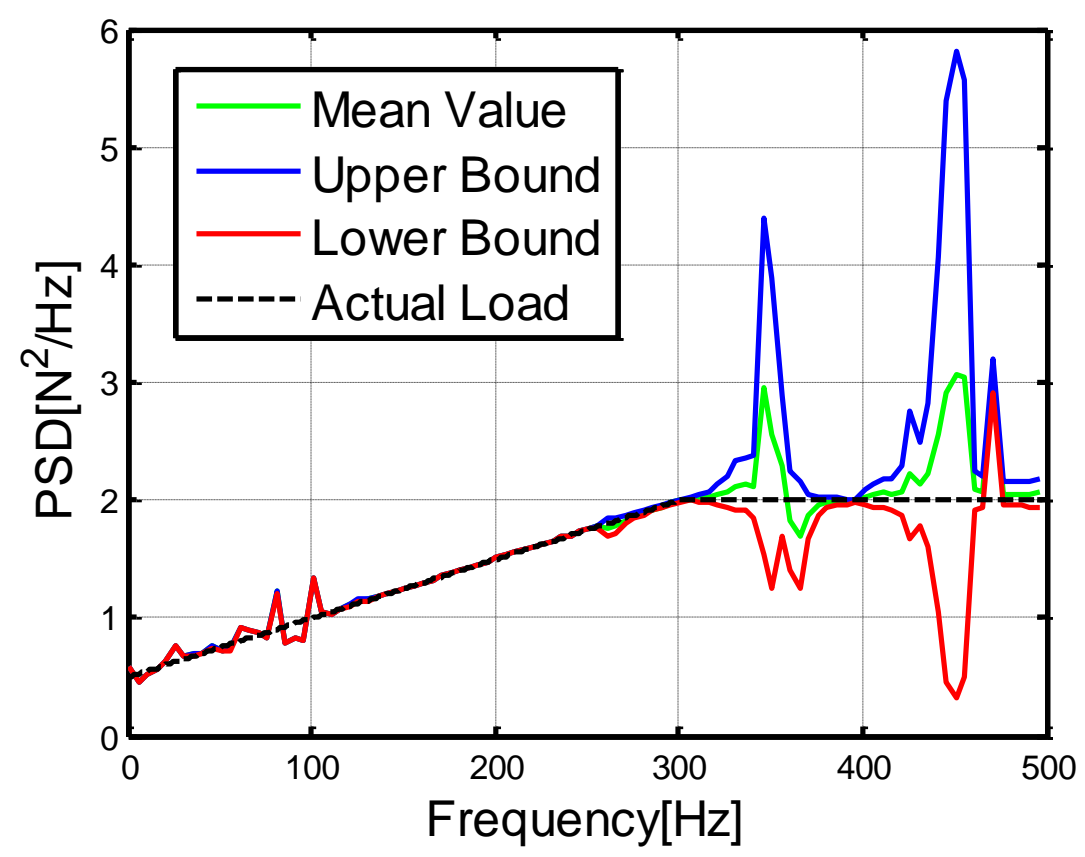

(c)

Fig. 9. The bounds of the random loads Auto-PSD computed by matrix perturbation method: (a) exciting position I ; (b) exciting position II ; (c) exciting position III. 


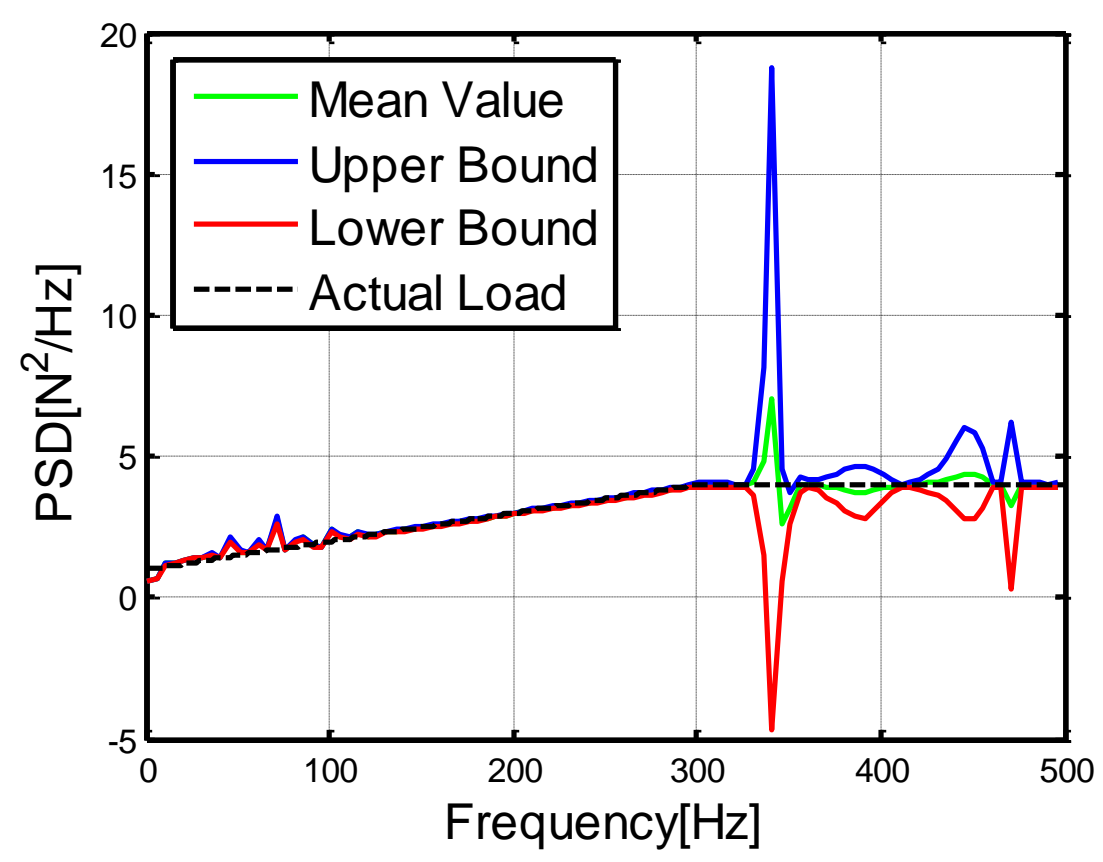

(a)

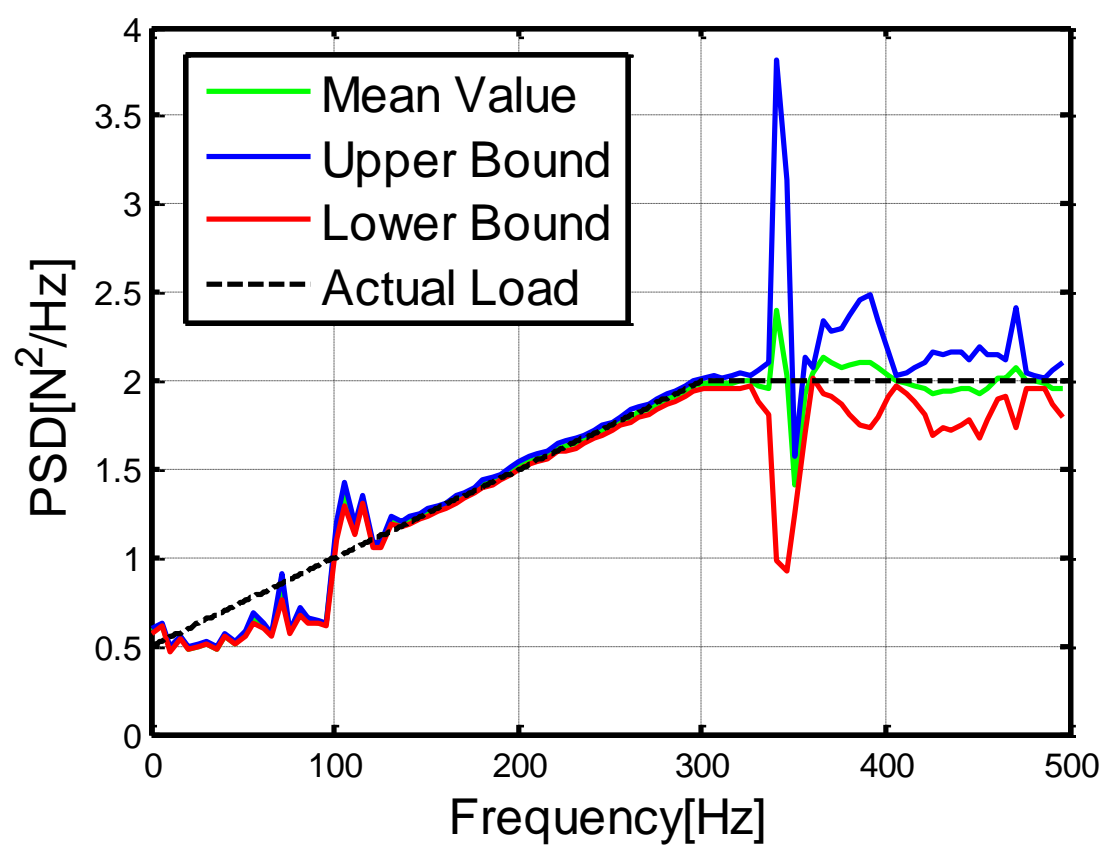

(b) 


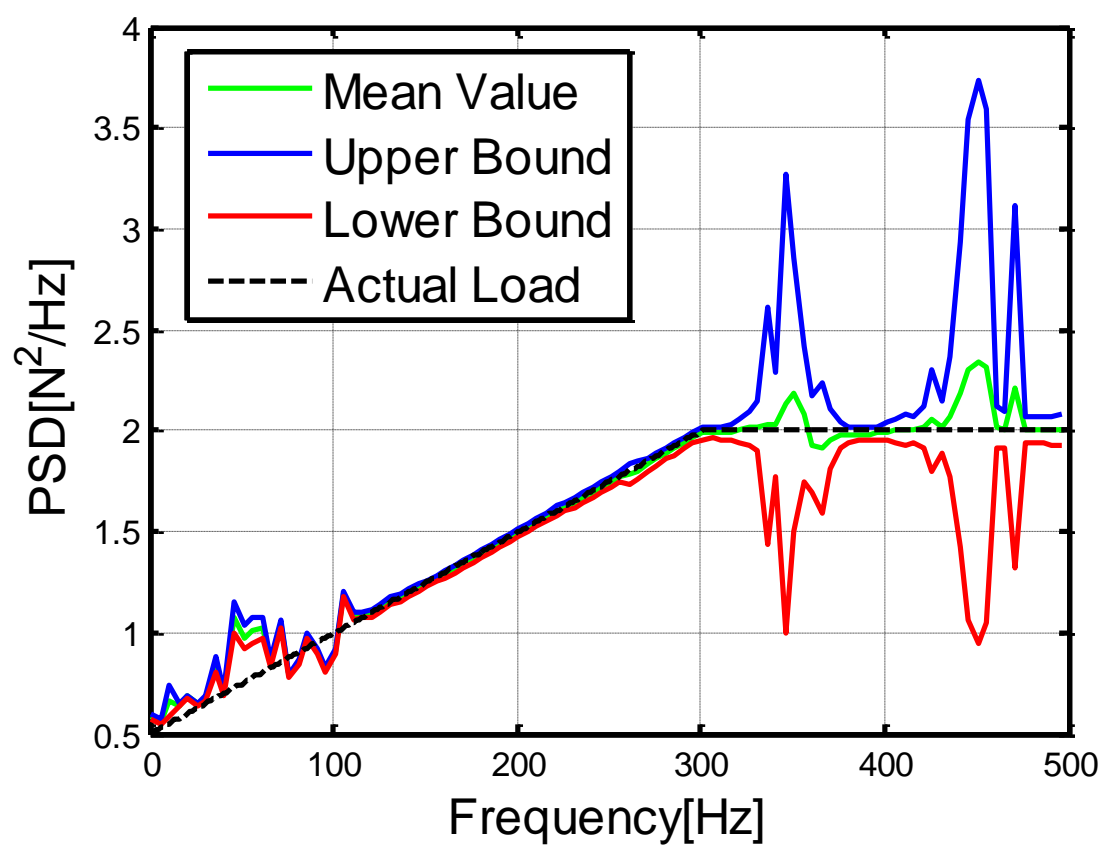

(c)

Fig. 10. The bounds of the random loads Auto-PSD computed by evidence theory: (a) exciting position I ; (b) exciting position II ; (c) exciting position III.

\subsection{A vehicle liftgate coupled with the interior cavity}

To further verify the effectiveness and superiority of the proposed improved regularization method on the random excitation identification compared with the other inverse methods, a numerical example is investigated on a vehicle liftgate coupled with the interior cavity. The automotive liftgate is the rear component of the vehicle body, which has a strong effect on the stiffness of the whole body. Owing to the coupling influence between the liftgate and the cavity, the vibration of the liftgate structure causes the acoustic noise in passenger compartment cavity.

As illustrated in Fig. 11, the simplified vehicle liftgate is mainly composed of glass, exterior plate, inner plate and several pieces of horizontal and vertical stiffener plates. The material properties of the liftgate plates are taken as follows: the material density is 7850 
$\mathrm{kg} / \mathrm{m}^{3}$, the elastic modulus is $210 \mathrm{GPa}$ and the damping loss factor is 0.06 . The material density, the elastic modulus and the structural damping loss factor of windows glass are $2500 \mathrm{~kg} / \mathrm{m}^{3}, 69 \mathrm{GPa}$ and 0.003 respectively. The acoustic part is made up of the passenger cavity containing the fluid air occupied by the seats. The properties of the passenger compartment cavity are set as follows: the sound velocity $v_{a}=340 \mathrm{~m} / \mathrm{s}$ and the mass density $\rho_{a}=1.2 \mathrm{~kg} / \mathrm{m}^{3}$. The four sides of the inner plate are fully constrained, and the back side of the acoustic cavity is considered to be coupled with the vibration of the structural liftgate. The exciting loads are imposed on three points of the liftgate exterior plate, which are selected randomly as the identifying random dynamic forces. The five acoustic pressure measuring points are located on the driver's ear and passenger's ear.

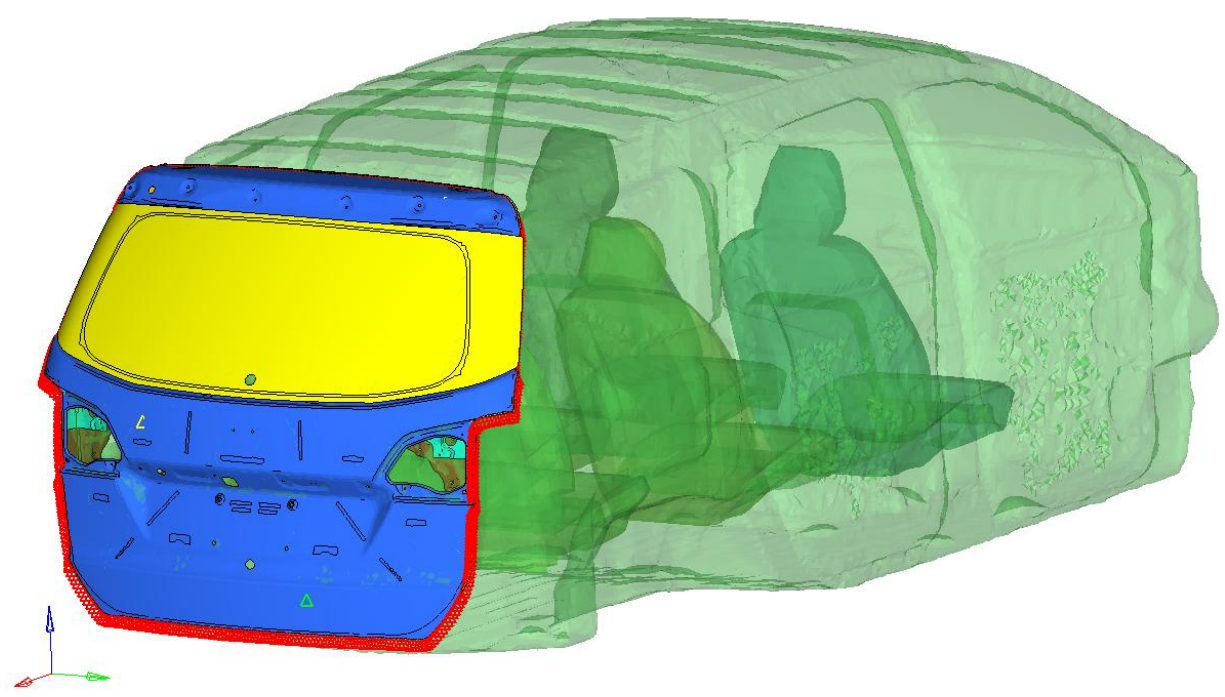

Fig. 11. The finite element model of vehicle liftgate and the coupled interior cavity.

We assume that the elastic modulus of the liftgate inner plate is a random parameter $\kappa$ following the three-peak PDF defined by Eq. (54). As illustrated in Fig. 12, the mean value and standard deviation of the PDF are $m u=7.819 \times 10^{3}, \quad \operatorname{std}(\kappa)=16.77$. 


$$
\begin{aligned}
& w(x)=a \frac{1}{\sqrt{2 \pi} \tau_{1}} \exp \left(-\frac{\left(x-\mu_{1}\right)^{2}}{2 \tau_{1}^{2}}\right)+b \frac{1}{\sqrt{2 \pi} \tau_{2}} \exp \left(-\frac{\left(x-\mu_{2}\right)^{2}}{2 \tau_{2}^{2}}\right) \\
& +(1-a-b) \frac{1}{\sqrt{2 \pi} \tau_{3}} \exp \left(-\frac{\left(x-\mu_{3}\right)^{2}}{2 \tau_{3}^{2}}\right)
\end{aligned}
$$

where $\mu_{1}=7.81 \times 10^{3}, \tau_{1}=3, \mu_{2}=7.83 \times 10^{3}, \tau_{2}=4, \mu_{3}=7.85 \times 10^{3}, \tau_{3}=4, a=0.2$, $b=0.6$.

Selecting a random value of $\kappa$, the random dynamic acoustic pressure responses are obtained based on finite element analysis. To simulate the actual measured responses, a random noise is added to the computational acoustic pressure as illustrated in Fig. 13.

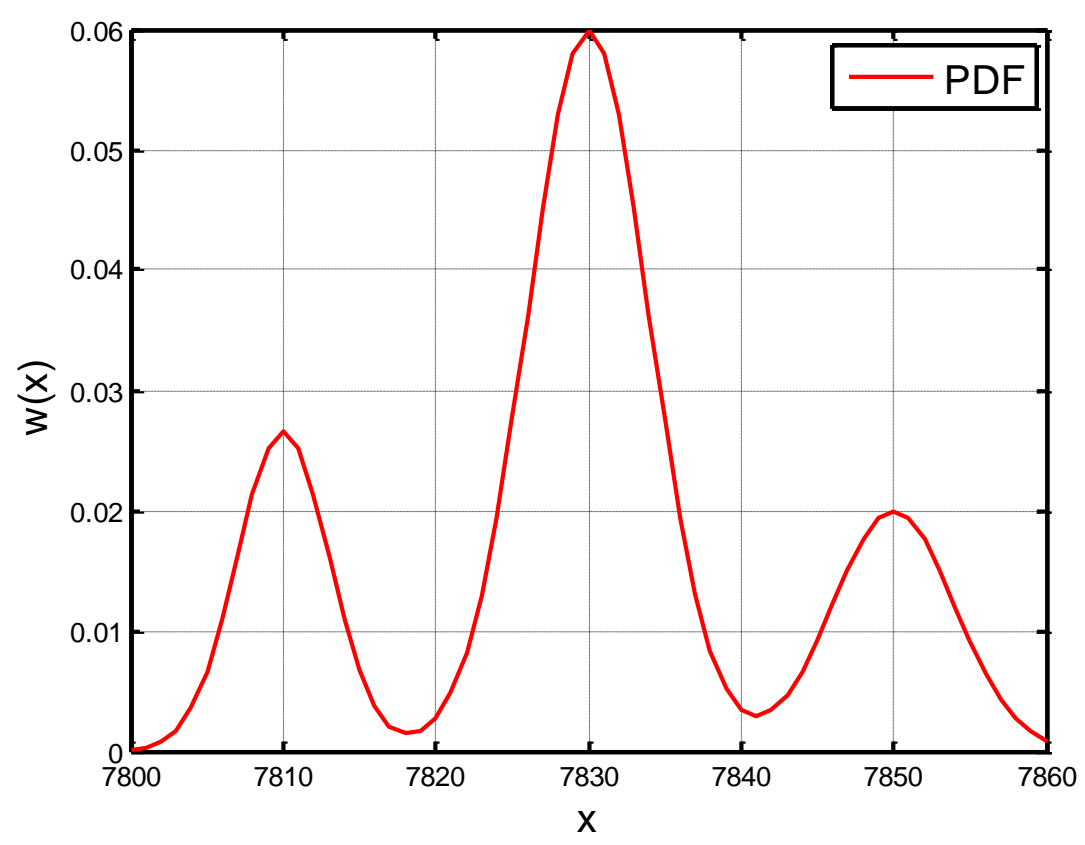

Fig. 12. The three-peak PDF of the random parameter $\kappa$. 


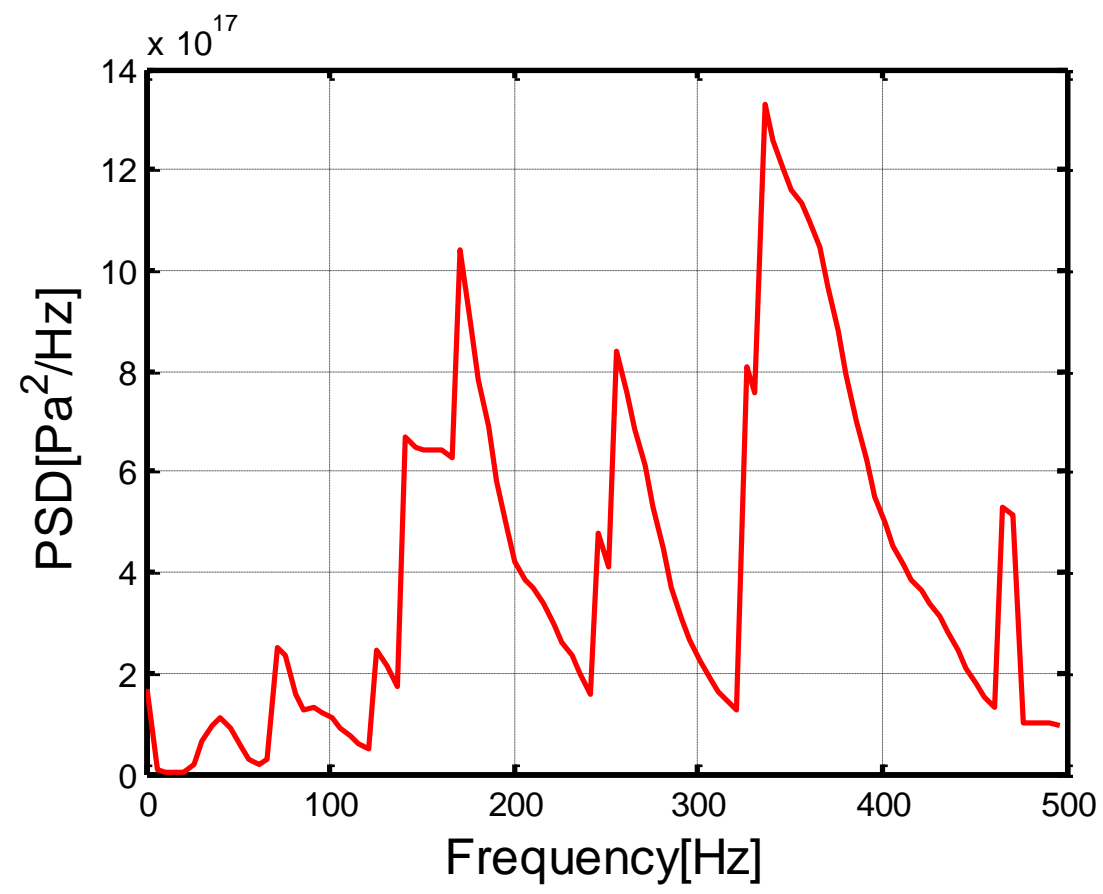

Fig. 13. Auto-PSD of the random acoustic pressure of response position 3 with $1 \%$ random noise.

Using the proposed improved regularization method and evidence analysis with three subintervals, the mean value and standard deviation of the random excitation to be identified are derived as shown in Fig. 14 and Fig. 15. Moreover, the bounds $\mathbf{S}_{\mathbf{F F}}^{\mathbf{L}}(\omega)$ and $\mathbf{S}_{\mathbf{F F}}^{\mathbf{U}}(\omega)$ shown in Fig. 16 are obtained based on the nominal mean value and nominal standard deviation. It is observed that the bounds obtained by evidence analysis mostly bracket the actual loads accurately and the mean value match the actual forces adequately in the full frequency range. Consequently, the proposed improved regularization method and evidence analysis method are verified to be accurate and effective in engineering application. 


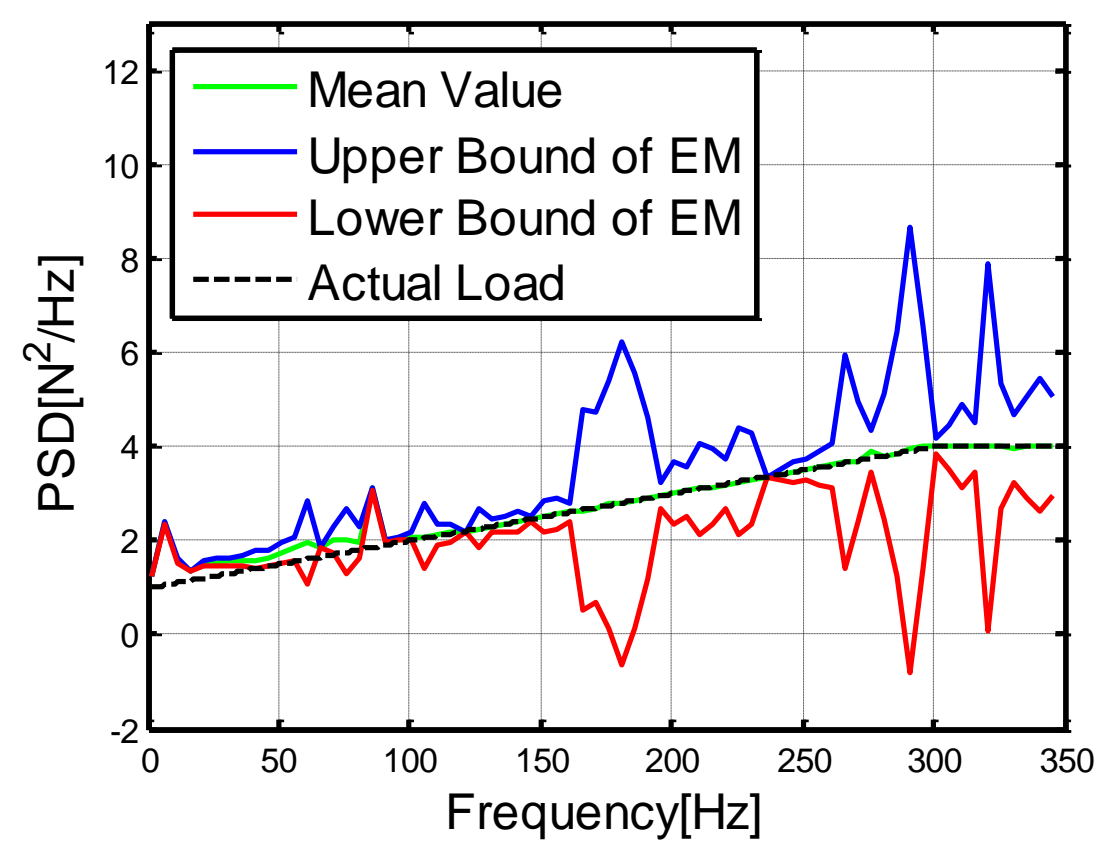

(a)

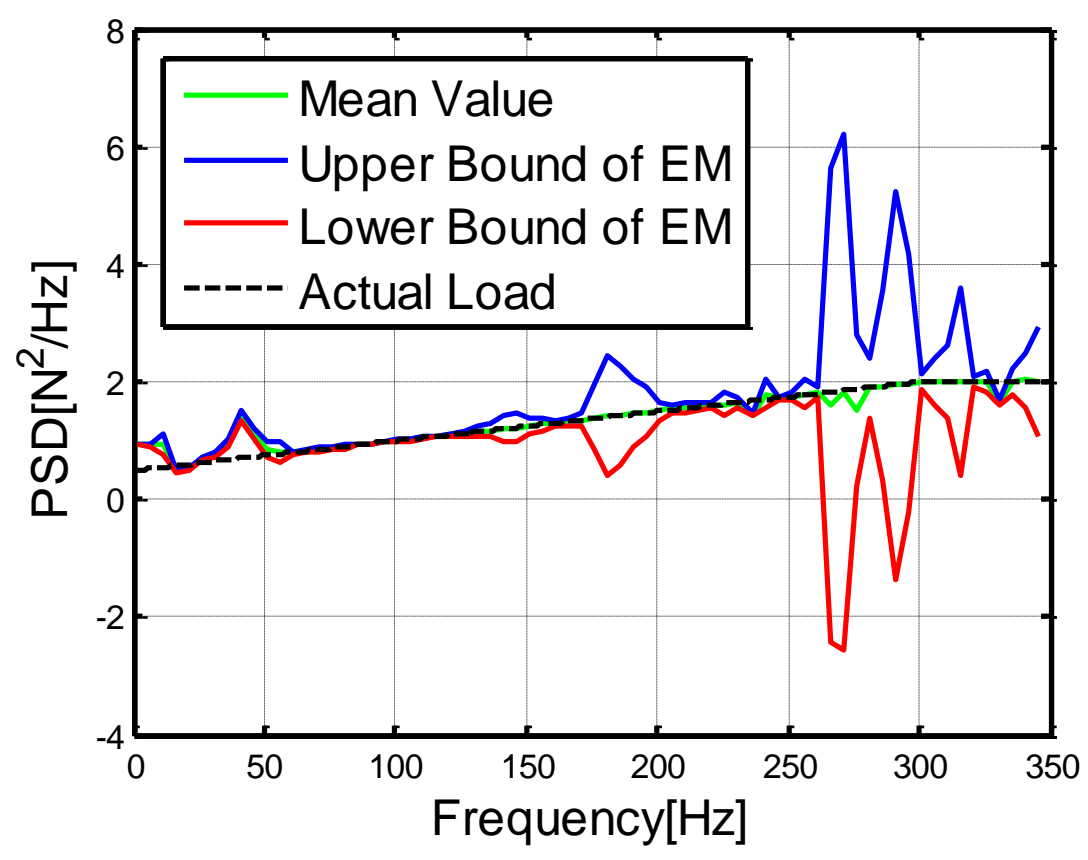

(b) 


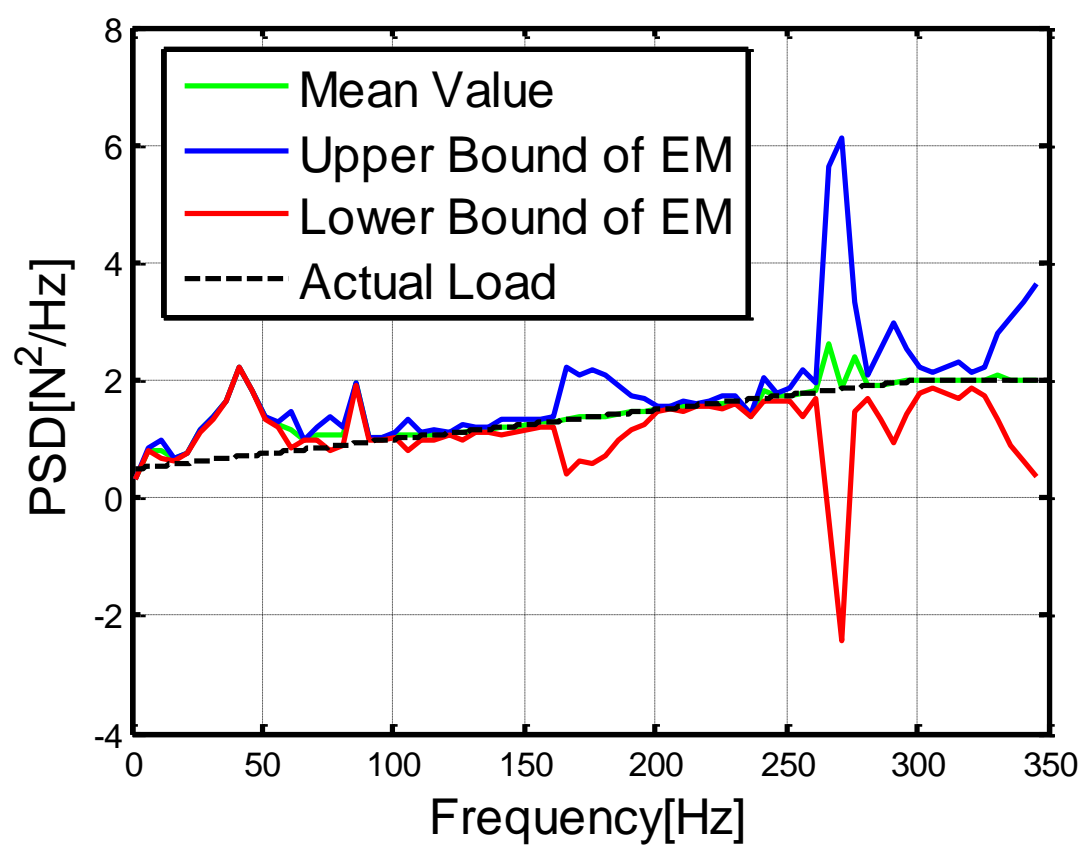

(c)

Fig. 14. The Auto-PSD mean value of random forces computed by the improved regularization method: (a) exciting position I ; (b) exciting position II ; (c) exciting position III.

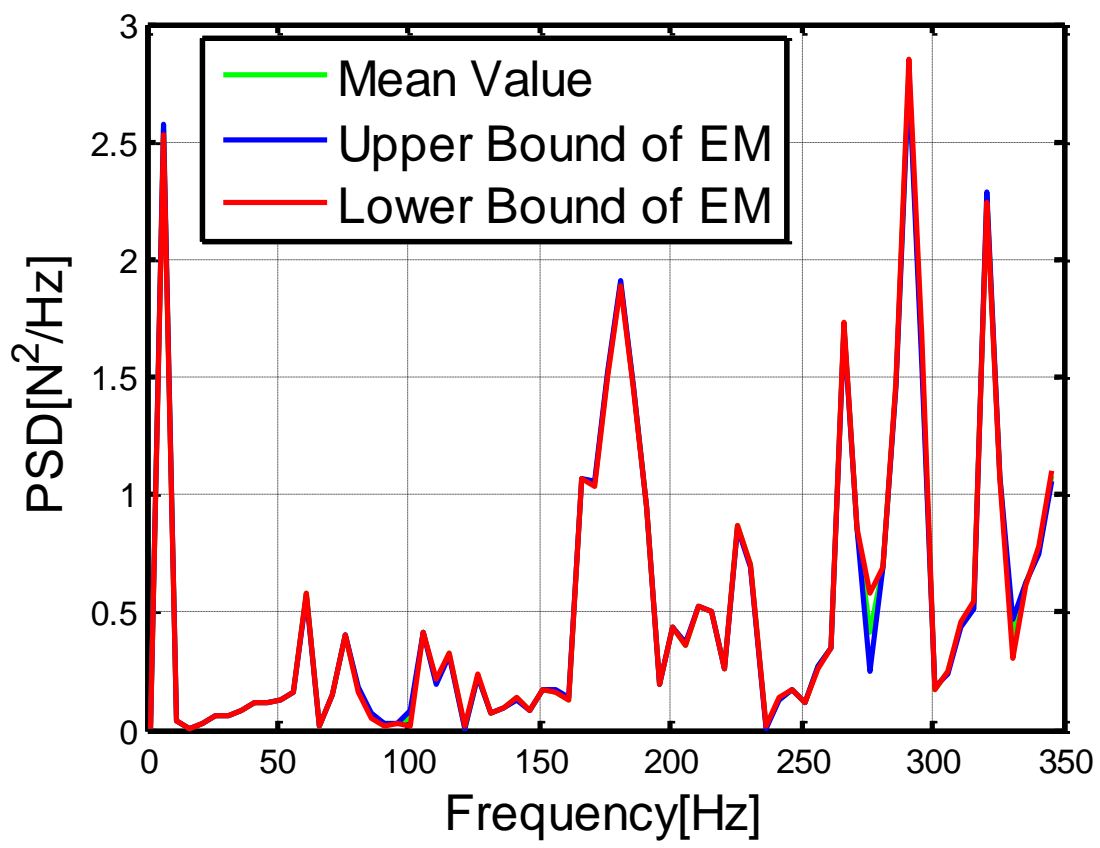

(a) 


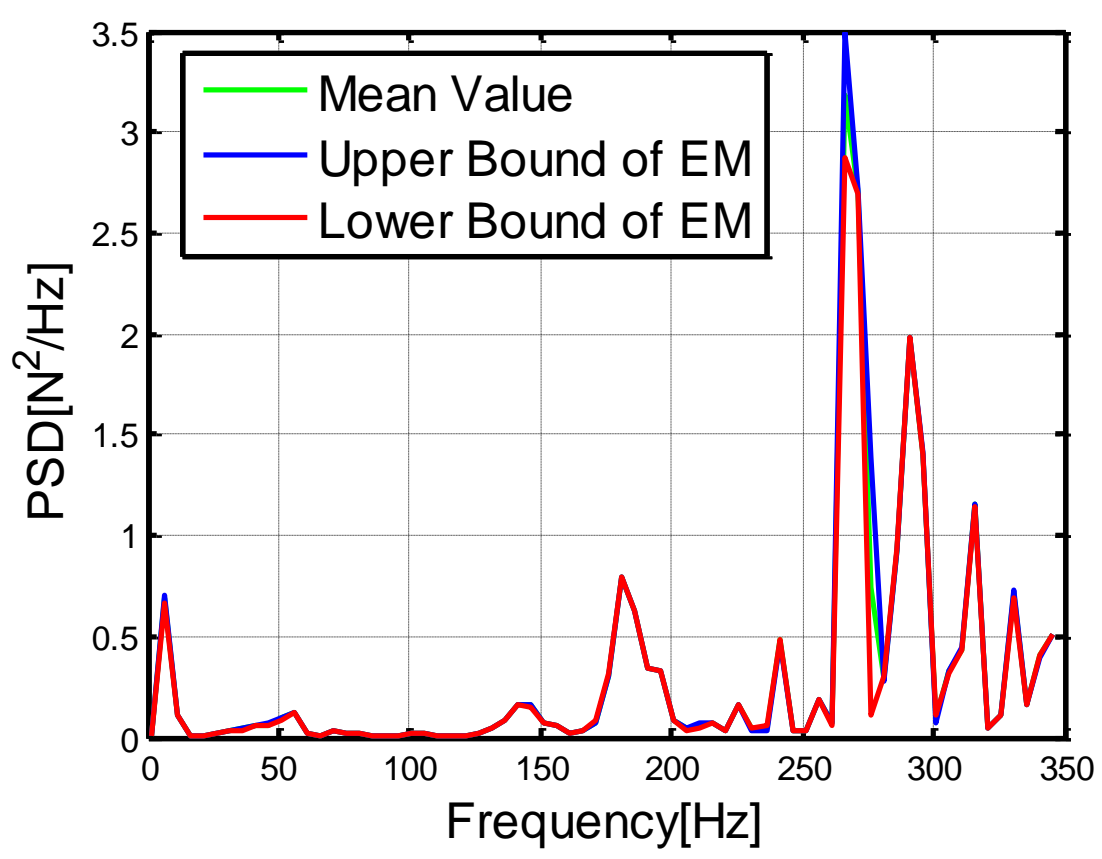

(b)

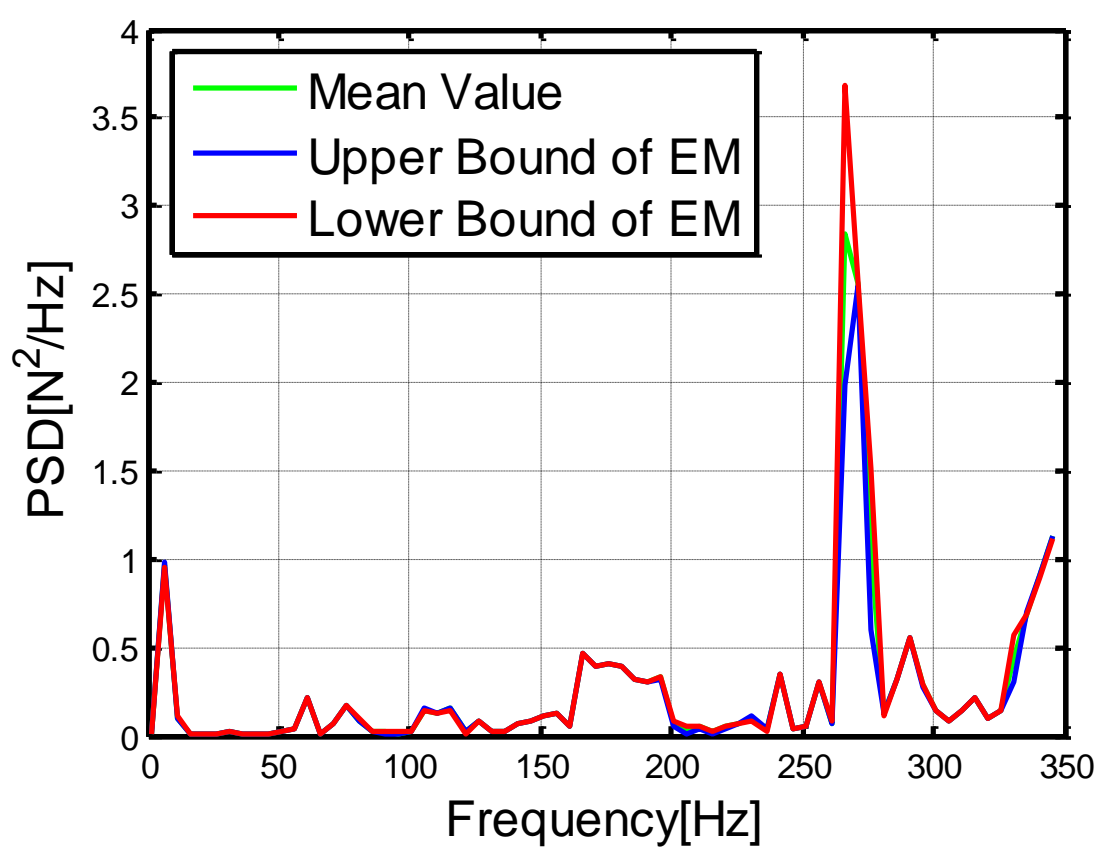

(c)

Fig. 15. The Auto-PSD standard deviation of random forces computed by the improved regularization method: (a) exciting position I ; (b) exciting position II ; (c) exciting position III. 


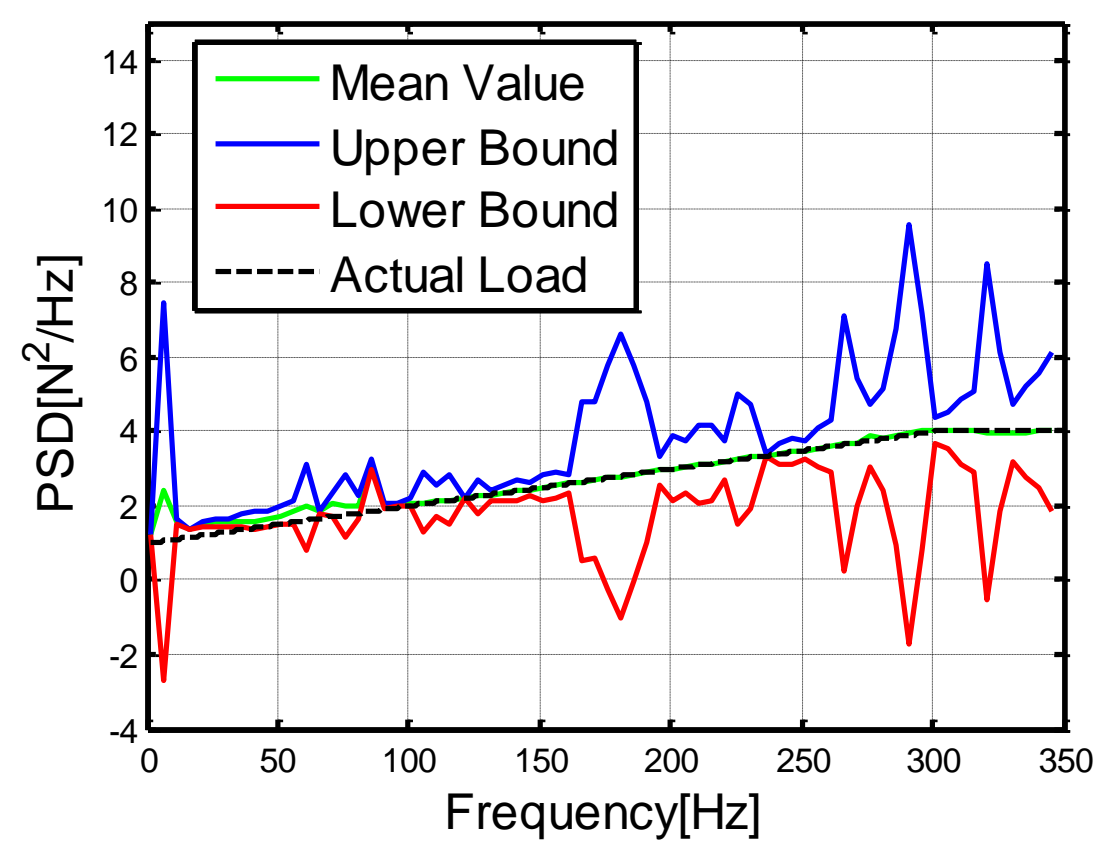

(a)

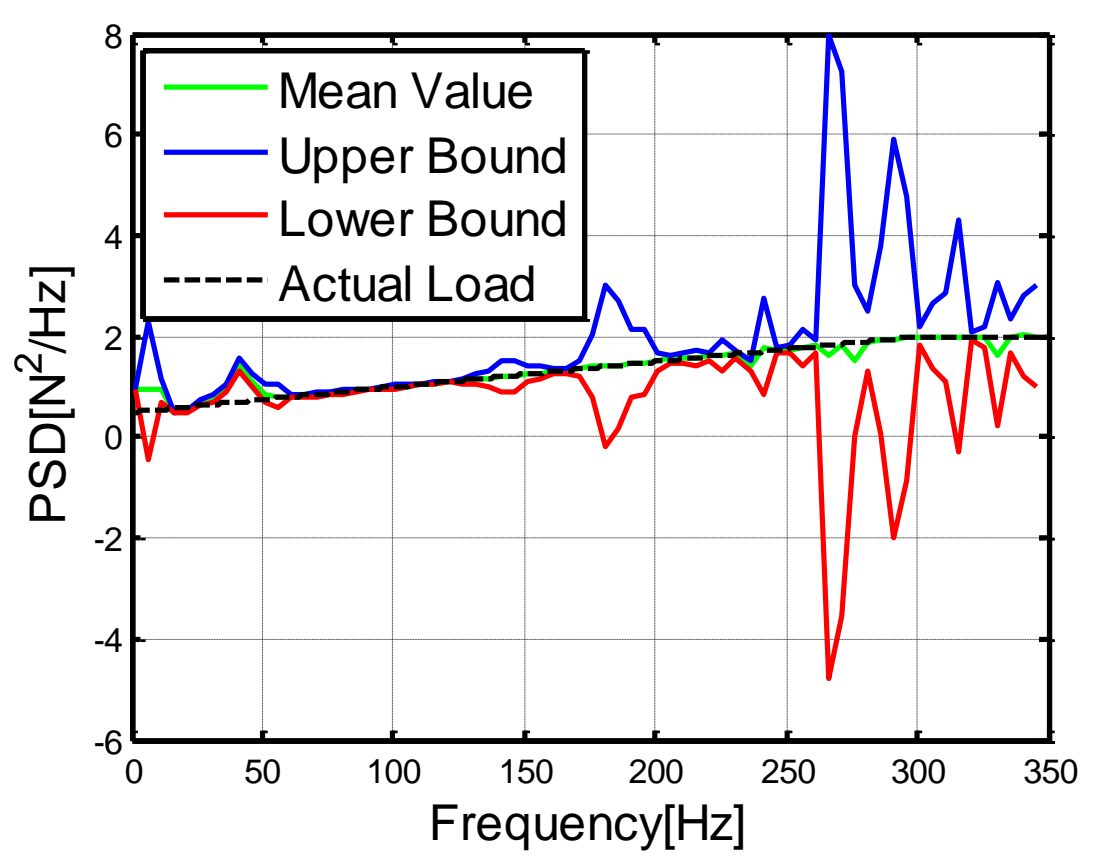

(b) 


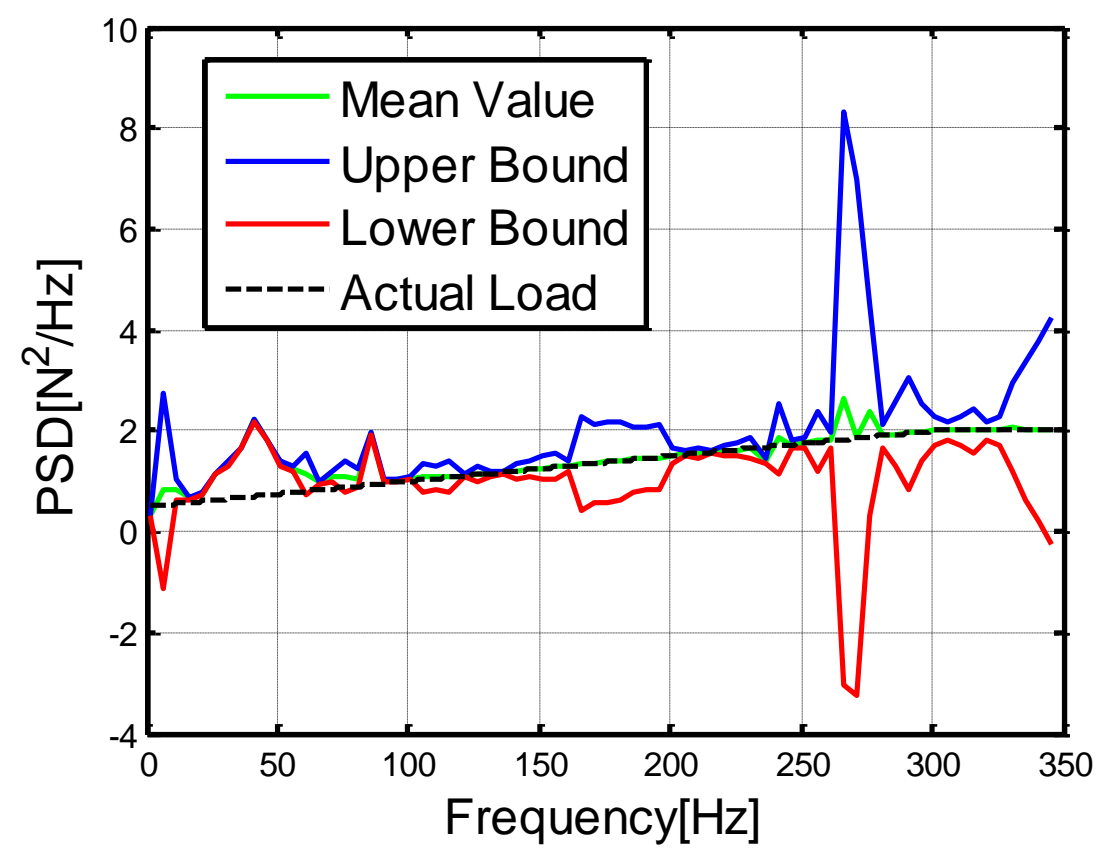

(c)

Fig. 16. The bounds of the random loads Auto-PSD computed by the improved regularization method: (a) exciting position I ; (b) exciting position II ; (c) exciting position III.

Additionally, to further verify the superiority of the proposed improved regularization method, the MPI method and Tikhonov regularization method are used to identify the random dynamic loads. Combined with the evidence theory, the bounds $\mathbf{S}_{\mathbf{F F}}^{\mathbf{L}}(\omega)$ and $\mathbf{S}_{\mathbf{F F}}^{\mathbf{U}}(\omega)$ shown in Fig. 17 are calculated using these two inverse methods. Through the comparison of the identified results as shown in Table 1, it is noted that the identified loads obtained by MPI method have a big error near low frequency. The improved regularization method reduces the identified error by $7.89 \times 10^{4} \mathrm{~N}^{2} / \mathrm{Hz}, 9.14 \times 10^{4} \mathrm{~N}^{2} / \mathrm{Hz}$ and $2.91 \times 10^{5}$ $\mathrm{N}^{2} / \mathrm{Hz}$ at $1 \mathrm{~Hz}, 4 \mathrm{~Hz}$ and $6 \mathrm{~Hz}$ respectively compared with the MPI method. As the condition number of FRF matrix in medium-high frequency region is small, the identified loads obtained by Tikhonov regularization method is too regularized, which brings an 
inaccurate result. The improved regularization method, by contrast, significantly decreases the error of identified loads and match the actual loads adequately in the full frequency range. Compared with the identified results by the Tikhonov regularization method as shown in Table 2, the improved regularization method decreases the identified error by $2.24 \times 10^{3}$ $\mathrm{N}^{2} / \mathrm{Hz}, 2.087 \mathrm{~N}^{2} / \mathrm{Hz}$ and $2.642 \mathrm{~N}^{2} / \mathrm{Hz}$ at $4 \mathrm{~Hz}, 225 \mathrm{~Hz}$ and $323 \mathrm{~Hz}$ respectively. Owing to the adaptive change of the improved regularization parameter along with the different order pseudo excitation and condition number of FRF matrix at different frequency, the proposed improved regularization method guarantees the stability of the identified forces near low frequency, and can prevent the over regularization of the identified loads in the case of small condition number. As shown in the figures and tables, PI stands for the Moore-Penrose pseudo inverse method, TR denotes the traditional Tikhonov regularization method, and IR represents the improved regularization method.

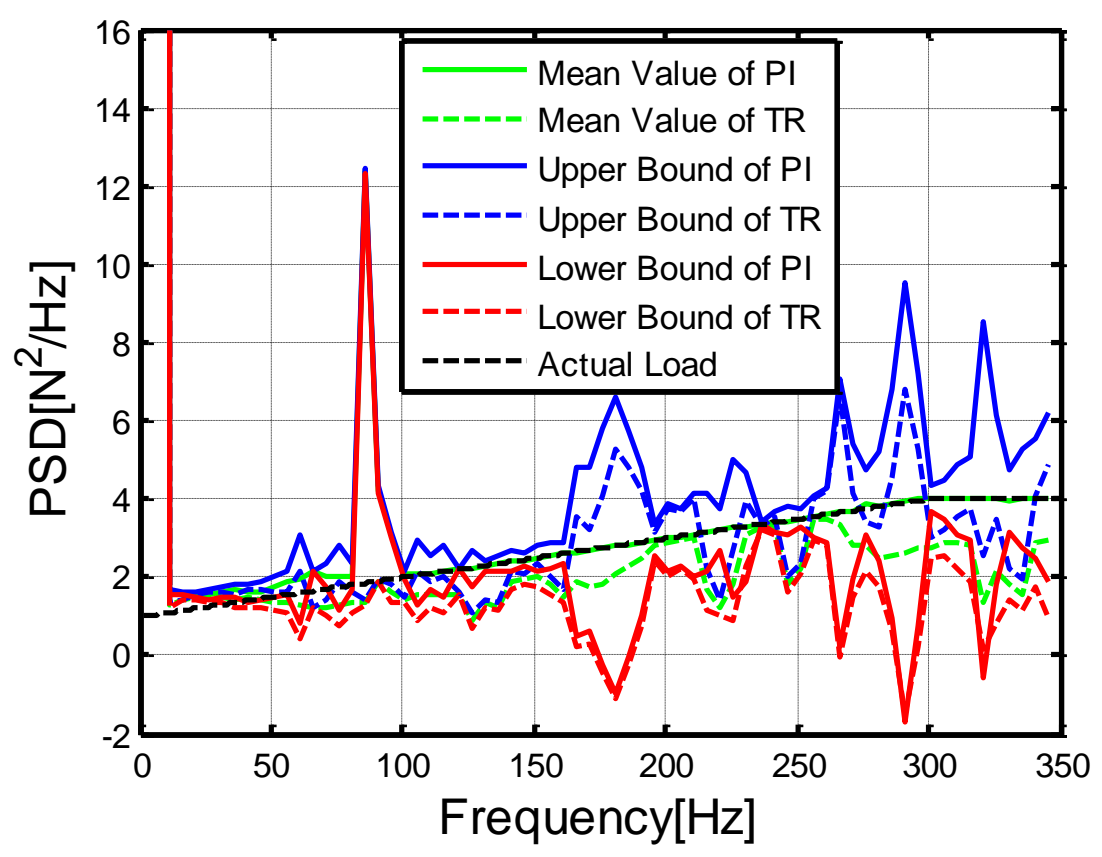

(a) 


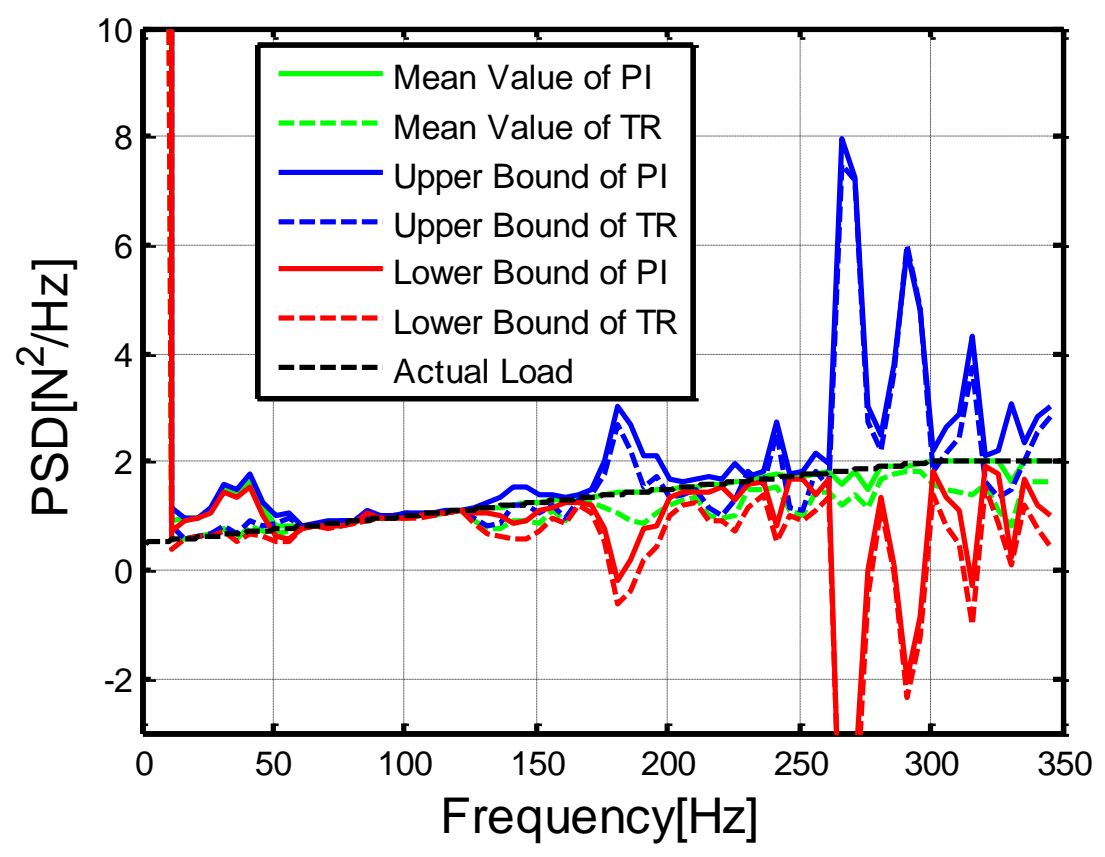

(b)

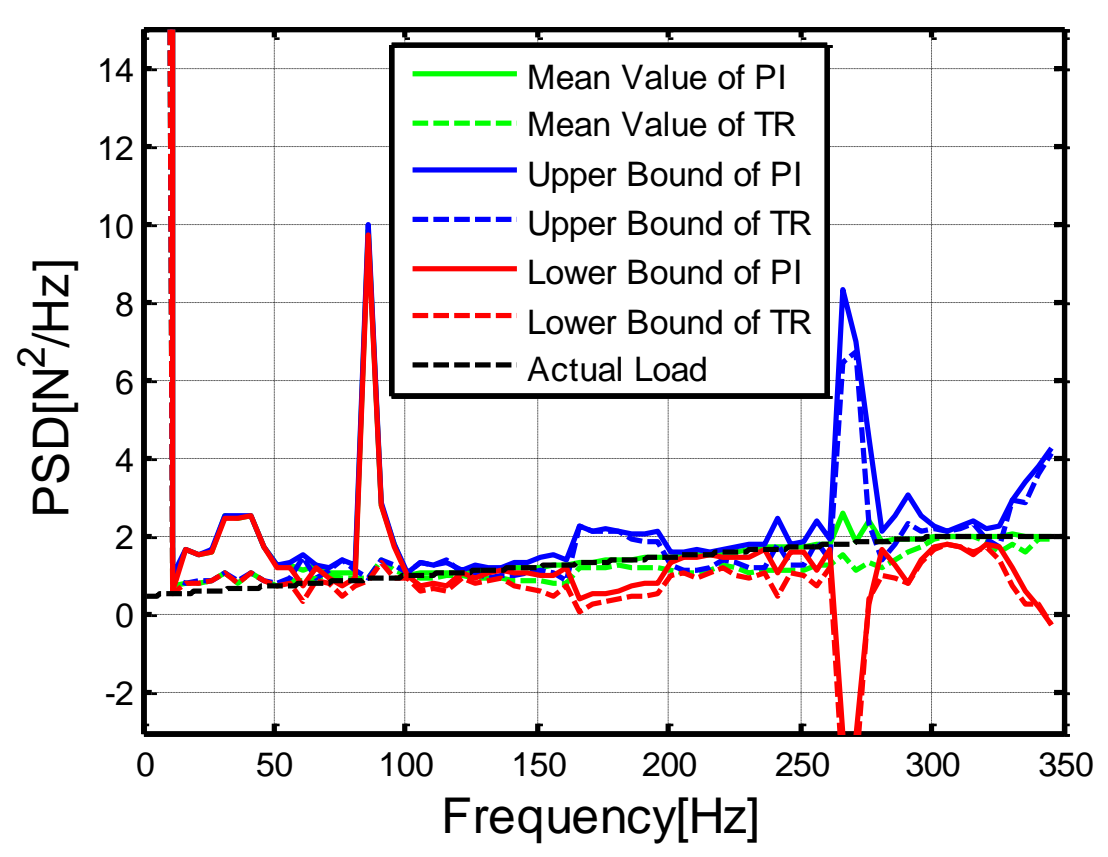

(c)

Fig. 17. The bounds of the random loads Auto-PSD computed by MPI method and Tikhonov regularization method: (a) exciting position I; (b) exciting position II; (c) exciting position III. 


\section{Table 1}

The Auto-PSD of random loads at exciting position III computed by MPI method and improved regularization method.

\begin{tabular}{ccccccc}
\hline Frequency & $1 \mathrm{~Hz}$ & $2 \mathrm{~Hz}$ & $4 \mathrm{~Hz}$ & $6 \mathrm{~Hz}$ & $87 \mathrm{~Hz}$ & $88 \mathrm{~Hz}$ \\
\hline Actual load & 0.500 & 0.505 & 0.515 & 0.525 & 0.930 & 0.935 \\
Mean value of PI & $7.89 \times 10^{4}$ & $4.43 \times 10^{4}$ & $9.14 \times 10^{4}$ & $2.91 \times 10^{5}$ & 13.38 & 13.38 \\
Mean value of IR & 0.323 & 0.371 & 0.582 & 0.814 & 1.007 & 1.011 \\
Reduced error & $7.89 \times 10^{4}$ & $4.43 \times 10^{4}$ & $9.14 \times 10^{4}$ & $2.91 \times 10^{5}$ & 12.38 & 12.38 \\
\hline
\end{tabular}

\section{Table 2}

The Auto-PSD of random loads at exciting position I computed by Tikhonov regularization method and improved regularization method.

\begin{tabular}{ccccccc}
\hline Frequency & $1 \mathrm{~Hz}$ & $4 \mathrm{~Hz}$ & $127 \mathrm{~Hz}$ & $225 \mathrm{~Hz}$ & $248 \mathrm{~Hz}$ & $323 \mathrm{~Hz}$ \\
\hline Actual load & 1.000 & 1.030 & 2.260 & 3.240 & 3.470 & 4.000 \\
Mean value of TR & 82.62 & $2.24 \times 10^{3}$ & 0.904 & 1.148 & 1.775 & 1.339 \\
Mean value of IR & 1.245 & 1.157 & 2.258 & 3.235 & 3.465 & 3.981 \\
Reduced error & 81.38 & $2.24 \times 10^{3}$ & 1.353 & 2.087 & 1.690 & 2.642 \\
\hline
\end{tabular}

\section{Conclusions}

In the present study, the random excitation identification problems for stochastic structural-acoustic coupled (SAC) system based on the sound pressure method are investigated in detail. The evidence theory is applied to solve the stochastic system problems combined with the interval analysis. An improved regularization method is proposed to handle the ill-posed problems in the process of inversion. With the validation on numerical examples, the major advantages of the proposed methods lay in:

a) The random dynamic load identification can be achieved based on the measured acoustic pressure responses instead of the structural vibration responses. The alternative sound pressure responses are measured in a non-contact way that is more accurate and convenient compared with the contact measurement.

b) With respect to the SAC system involving random parameter with large coefficient 
of variation and multimodal distribution, the proposed method based on evidence theory and interval analysis is able to identify the unknown random excitation more effectively and accurately compared with the matrix perturbation method.

c) Due to the adaptive of the constructed regularization parameter along with the different order pseudo excitation and condition number of frequency response function (FRF) matrix at different frequency, the proposed improved regularization method guarantees the stability of the identified forces near low frequency, and can prevent the over regularization of the identified loads in the case of small condition number.

d) The improved regularization method reduces the identified error by $7.89 \times 10^{4} \mathrm{~N}^{2} / \mathrm{Hz}$, $9.14 \times 10^{4} \mathrm{~N}^{2} / \mathrm{Hz}$ and $2.91 \times 10^{5} \mathrm{~N}^{2} / \mathrm{Hz}$ at $1 \mathrm{~Hz}, 4 \mathrm{~Hz}$ and $6 \mathrm{~Hz}$ respectively compared with the MPI method, and decreases the identified error by $2.24 \times 10^{3} \mathrm{~N}^{2} / \mathrm{Hz}$, $2.087 \mathrm{~N}^{2} / \mathrm{Hz}$ and $2.642 \mathrm{~N}^{2} / \mathrm{Hz}$ at $4 \mathrm{~Hz}, 225 \mathrm{~Hz}$ and $323 \mathrm{~Hz}$ respectively compared with the Tikhonov regularization method.

e) For the stochastic system with large uncertainties, the identified excitation region using the first-order Taylor analysis would fail to contain the real region of the dynamic load. In this study, the evidence theory is combined with interval analysis. By discretizing the PDF of each random parameter into several subintervals, the variations of random parameters in each subinterval is reduced, which enhances the assumption of first-order Taylor expansion. 


\section{Acknowledgements}

The project is supported by the Foundation for Innovative Research Groups of the National Natural Science Foundation of China (Grant No. 51621004).

\section{Appendix A}

Eq. (13) can be rewritten as

$$
\begin{aligned}
\tilde{\mathbf{P}}_{j}(\omega)+\Delta \tilde{\mathbf{P}}_{j}(\omega) & =\mathbf{T}(\omega)\left(\tilde{\mathbf{F}}_{j}(\omega)+\Delta \tilde{\mathbf{F}}_{j}(\omega)\right) \\
\tilde{\mathbf{P}}_{j}(\omega) & =\mathbf{T}(\omega) \tilde{\mathbf{F}}_{j}(\omega)
\end{aligned}
$$

Combining with Eq. (B.2), Eq. (B.1) is transformed into

$$
\Delta \tilde{\mathbf{P}}_{j}(\omega)=\mathbf{T}(\omega) \Delta \tilde{\mathbf{F}}_{j}(\omega)
$$

Consequently, the error terms of identified pseudo force can be described as

$$
\Delta \tilde{\mathbf{F}}_{j}(\omega)=\mathbf{T}(\omega)^{+} \Delta \tilde{\mathbf{P}}_{j}(\omega)
$$

in which the superscript + represents the generalized inversion. To determine the relative error of identifying pseudo force, the matrix norm is adopted to Eq. (B.2) and Eq. (B.4):

$$
\begin{gathered}
\left\|\tilde{\mathbf{P}}_{j}(\omega)\right\| \leq\|\mathbf{T}(\omega)\|\left\|\tilde{\mathbf{F}}_{j}(\omega)\right\| \\
\left\|\Delta \tilde{\mathbf{F}}_{j}(\omega)\right\| \leq\left\|\mathbf{T}(\omega)^{+}\right\|\left\|\Delta \tilde{\mathbf{P}}_{j}(\omega)\right\|
\end{gathered}
$$

Multiplying Eq. (B.6) by Eq. (B.5):

$$
\left\|\Delta \tilde{\mathbf{F}}_{j}(\omega)\right\|\left\|\tilde{\mathbf{P}}_{j}(\omega)\right\| \leq\left\|\mathbf{T}(\omega)^{+}\right\|\left\|\Delta \tilde{\mathbf{P}}_{j}(\omega)\right\|\|\mathbf{T}(\omega)\|\left\|\tilde{\mathbf{F}}_{j}(\omega)\right\|
$$

Hence, the relative error of identifying pseudo excitations can be derived

$$
\frac{\left\|\Delta \tilde{\mathbf{F}}_{j}(\omega)\right\|}{\left\|\tilde{\mathbf{F}}_{j}(\omega)\right\|} \leq\left\|\mathbf{T}(\omega)^{+}\right\|\|\mathbf{T}(\omega)\| \frac{\left\|\Delta \tilde{\mathbf{P}}_{j}(\omega)\right\|}{\left\|\tilde{\mathbf{P}}_{j}(\omega)\right\|}
$$

Eq. (B.8) can be rewritten as

$$
\frac{\left\|\Delta \tilde{\mathbf{F}}_{j}(\omega)\right\|}{\left\|\tilde{\mathbf{F}}_{j}(\omega)\right\|} \leq \operatorname{cond}(\mathbf{T}(\omega)) \frac{\left\|\Delta \tilde{\mathbf{P}}_{j}(\omega)\right\|}{\left\|\tilde{\mathbf{P}}_{j}(\omega)\right\|}
$$


where the condition number of FRF $\operatorname{cond}(\mathbf{T}(\omega))=\left\|\mathbf{T}(\omega)^{+}\right\|\|\mathbf{T}(\omega)\|$.

\section{References}

[1] L. Ding, H. Hao, X. Zhu, Evaluation of dynamic vehicle axle loads on bridges with different surface conditions, Journal of Sound \& Vibration, 323 (2016) 826-848.

[2] R.H. Scanlan, The action of flexible bridges under wind, II: Buffeting theory, Journal of Sound \& Vibration, 60 (1978) 201-211.

[3] A. Alvandi, C. Cremona, Assessment of vibration-based damage identification techniques, Journal of Sound \& Vibration, 292 (2006) 179-202.

[4] R. Rackwitz, B. Flessler, Structural reliability under combined random load sequences, Computers \& Structures, 9 (1978) 489-494.

[5] A.N. Thite, D.J. Thompson, The quantification of structure-borne transmission paths by inverse methods. Part 1: Improved singular value rejection methods, Journal of Sound \& Vibration, 264 (2003) 411-431.

[6] A.N. Thite, D.J. Thompson, The quantification of structure-borne transmission paths by inverse methods. Part 2: Use of regularization techniques, Journal of Sound \& Vibration, 264 (2003) 433-451.

[7] Y.E. Lage, N.M.M. Maia, M.M. Neves, A.M.R. Ribeiro, Force identification using the concept of displacement transmissibility, Journal of Sound \& Vibration, 332 (2013) 1674-1686.

[8] Z.R. Lu, S.S. Law, Identification of system parameters and input force from output only, Mechanical Systems \& Signal Processing, 21 (2007) 2099-2111.

[9] J. Liu, X. Sun, X. Han, C. Jiang, D. Yu, A novel computational inverse technique for load identification using the shape function method of moving least square fitting, Computers \& Structures, 144 (2014) 127-137.

[10] F.D.B. Jr, W.G. Flannelly, Model Verification of Force Determination for Measuring Vibratory Loads, Journal of the American Helicopter Society, 24 (1979) 10-18.

[11] S.E.S. Karlsson, IDENTIFICATION OF EXTERNAL STRUCTURAL LOADS FROM MEASURED HARMONIC RESPONSES, Journal of Sound \& Vibration, 196 (1996) 59-74.

[12] G.R. Liu, W.B. Ma, X. Han, An inverse procedure for identification of loads on composite laminates, Composites Part B, 33 (2002) 425-432.

[13] V. Arora, Y.H. Wijnant, A.D. Boer, Acoustic-based damage detection method, Applied Acoustics, 80 (2014) 23-27.

[14] Q. Wan, W.K. Jiang, Near field acoustic holography (NAH) theory for cyclostationary sound field and its application, Journal of Sound \& Vibration, 290 (2006) 956-967.

[15] J.D. Maynard, E.G. Williams, Y. Lee, Nearfield acoustic holography: I. Theory of generalized holography and the development of NAH, Jasa, 78 (1985) 1395-1413.

[16] X. Zhao, S.F. Wu, Reconstruction of vibro-acoustic fields using hybrid nearfield acoustic holography, Journal of Sound \& Vibration, 282 (2005) 1183-1199.

[17] C.M. Lee, Y. Xu, A modified transfer matrix method for prediction of transmission loss of multilayer acoustic materials, Journal of Sound \& Vibration, 326 (2009) 290-301.

[18] J.H. Deng, C.S. Gu, X.D. Liu, H.G. Li, Sound source identification and contribution analysis based on acoustic array technology, Journal of Vibration Engineering, (2010).

[19] S. Chakraborty, S.S. Dey, A stochastic finite element dynamic analysis of structures with uncertain parameters, International Journal of Mechanical Sciences, 40 (1998) 1071-1087.

[20] G.I. Schuëller, A state-of-the-art report on computational stochastic mechanics, Probabilistic Engineering 
Mechanics, 12 (1997) 197-321.

[21] J. Liu, X. Sun, X. Han, C. Jiang, D. Yu, Dynamic load identification for stochastic structures based on Gegenbauer polynomial approximation and regularization method, Mechanical Systems \& Signal Processing, $\mathrm{s}$ 56-57 (2015) 35-54.

[22] X. Sun, Identification Method of Dynamic Loads for Stochastic Structures Based on Matrix Perturbation Theory, Journal of Mechanical Engineering, 50 (2014) 148.

[23] A. Chatterjee, Sensitivity and Error Analysis of a Coupled Micro-Resonator Array for Ultra-Sensitive Mass Detection Using Matrix Perturbation Theory, IEEE Sensors Journal, 16 (2016) 1304-1309.

[24] W.K. Liu, T. Belytschko, A. Mani, Probabilistic finite elements for nonlinear structural dynamics, Computer Methods in Applied Mechanics \& Engineering, 56 (1986) 61-81.

[25] C.L. Wu, X.P. Ma, T. Fang, A complementary note on Gegenbauer polynomial approximation for random response problem of stochastic structure, Probabilistic Engineering Mechanics, 21 (2006) 410-419.

[26] S.G. Ghangrekar, Application of gegenbauer polynomial approximation to small transverse oscillations of string, Proceedings of the IEEE, 9 (1976) 471-472.

[27] A.P. Dempster, N.M. Laird, D.B. Rubin, Maximum Likelihood from Incomplete Data via the EM Algorithm, Journal of the Royal Statistical Society, 39 (1977) 1-38.

[28] JamesInglis, A Mathematical Theory of Evidence, Technometrics, 20 (1976) 106-106.

[29] M.J. Zhou, A Design Optimization Method Using Evidence Theory, Journal of Mechanical Design, 128 (2006) 1153-1161.

[30] H. Agarwal, J.E. Renaud, E.L. Preston, D. Padmanabhan, Uncertainty quantification using evidence theory in multidisciplinary design optimization, Reliability Engineering \& System Safety, 85 (2004) 281-294.

[31] E. Alyanak, R. Grandhi, H.R. Bae, Gradient projection for reliability-based design optimization using evidence theory, Engineering Optimization, 40 (2008) 923-935.

[32] Y.C. Bai, X. Han, C. Jiang, J. Liu, Comparative study of metamodeling techniques for reliability analysis using evidence theory, Advances in Engineering Software, 53 (2012) 61-71.

[33] Y.C. Bai, C. Jiang, X. Han, D.A. Hu, Evidence-theory-based structural static and dynamic response analysis under epistemic uncertainties, Finite Elements in Analysis \& Design, 68 (2013) 52-62.

[34] C. Jiang, Z. Zhang, X. Han, J. Liu, A novel evidence-theory-based reliability analysis method for structures with epistemic uncertainty, Computers \& Structures, 129 (2013) 1-12.

[35] C. Jiang, X. Han, G.Y. Lu, J. Liu, Z. Zhang, Y.C. Bai, Correlation analysis of non-probabilistic convex model and corresponding structural reliability technique, Computer Methods in Applied Mechanics \& Engineering, 200 (2011) 2528-2546.

[36] C. Jiang, R.G. Bi, G.Y. Lu, X. Han, Structural reliability analysis using non-probabilistic convex model, Computer Methods in Applied Mechanics \& Engineering, 254 (2013) 83-98.

[37] N. Okubo, S. Tanabe, T. Tatsuno, Identification of Forces Generated by a Machine Under Operating Condition, (1985).

[38] X. Sun, L. Jie, H. Xu, J. Chao, C. Rui, A new improved regularization method for dynamic load identification, Inverse Problems in Science \& Engineering, 22 (2014) 1062-1076.

[39] Y. Liu, W.S.S. Jr, Dynamic force identification based on enhanced least squares and total least-squares schemes in the frequency domain, Journal of Sound \& Vibration, 282 (2005) 37-60.

[40] M. Aucejo, O.D. Smet, A multiplicative regularization for force reconstruction, Mechanical Systems \& Signal Processing, 85 (2017) 730-745.

[41] M. Aucejo, O.D. Smet, A space-frequency multiplicative regularization for force reconstruction problems, Mechanical Systems \& Signal Processing, 104 (2018). 
[42] J. Liu, X. Meng, D. Zhang, C. Jiang, X. Han, An efficient method to reduce ill-posedness for structural dynamic load identification, Mechanical Systems \& Signal Processing, 95 (2017) 273-285.

[43] E. Zhang, J. Antoni, P. Feissel, Bayesian force reconstruction with an uncertain model, Journal of Sound \& Vibration, 331 (2012) 798-814.

[44] Y. Jia, Z. Yang, N. Guo, L. Wang, Random dynamic load identification based on error analysis and weighted total least squares method, Journal of Sound \& Vibration, 358 (2015) 111-123.

[45] Y. Jia, Z. Yang, Q. Song, Experimental study of random dynamic loads identification based on weighted regularization method, Journal of Sound \& Vibration, 342 (2015) 113-123.

[46] J. Lin, W. Zhang, J. Li, Structural responses to arbitrarily coherent stationary random excitations, Computers \& Structures, 50 (1994) 629-633.

[47] S. Yin, D. Yu, H. Yin, B. Xia, Interval and random analysis for structure-acoustic systems with large uncertain-but-bounded parameters, Computer Methods in Applied Mechanics \& Engineering, 305 (2016) 910-935.

[48] J.H. Lin, X.L. Guo, H. Zhi, W.P. Howson, F.W. Williams, Computer simulation of structural random loading identification, Computers \& Structures, 79 (2001) 375-387.

[49] A.N. Thite, D.J. Thompson, Selection of response measurement locations to improve inverse force determination, Applied Acoustics, 67 (2006) 797-818.

[50] P.C. Hansen, REGULARIZATION TOOLS: A Matlab package for analysis and solution of discrete ill-posed problems, Numerical Algorithms, 6 (1994) 1-35.

[51] G.H. Golub, C. Reinsch, Singular Value Decomposition and Least Squares Solutions, Springer Berlin Heidelberg, 1971. 